\title{
Study of $B_{c} \rightarrow D S$ decays in the perturbative QCD approach
}

\author{
Zhi-Tian Zou and Ying $\mathrm{Li}^{*}$ \\ Department of Physics, Yantai University, Yantai 264005, China \\ Xin Liu \\ School of Physics and Electronic Engineering, Jiangsu Normal University, Xuzhou 221116, China
}

(Received 8 December 2017; published 23 March 2018)

\begin{abstract}
Within the framework of the perturbative QCD approach based on $k_{T}$ factorization, we study 40 $B_{c} \rightarrow D S$ decay modes in the leading order and leading power, where " $S$ " stands for the light scalar meson. Under two different scenarios (S1 and S2) for the description of scalar mesons, we explore the branching fractions and related $C P$ asymmetries. As a heavy meson consisting of two heavy quarks with different flavor, the light-cone distribution amplitude of the $B_{c}$ meson has not been well defined, and therefore the $\delta$ function is adopted. We find that the contributions of emission diagrams are suppressed by the vector decay constants and Cabibbo-Kobayashi-Maskawa elements, and the contributions of annihilation are dominant. After the calculation, we also find that some branching fractions are in the range $\left[10^{-5}, 10^{-4}\right]$, which could be measured at the current LHCb experiment, and other decays with smaller branching fractions will be tested at high-energy colliders in the future. Furthermore, some decay modes have large $C P$ asymmetries, but they are unmeasurable currently due to the small branching fractions.
\end{abstract}

DOI: 10.1103/PhysRevD.97.053005

\section{INTRODUCTION}

The study of weak decays of the $B_{c}$ meson are of interest, since it is the only heavy meson consisting of two heavy quarks with different flavors. The Collider Detector at Fermilab (CDF) Collaboration reported the discovery of the $B_{c}$ ground state in $p \bar{p}$ collisions [1], which was further confirmed by the CDF and D0 Collaborations [2] with more precise measurements. Currently, with high collision energy and high luminosity, the Large Hadron Collider (LHC) could collect about $10^{9} B_{c}$ meson events each year [3]. Based on such large samples, many weak decay modes of the $B_{c}$ meson have been measured by the LHCb Collaboration [4].

In the quark model, the $B_{c}^{+}$meson is the lowest-lying bound state of a bottom antiquark and a charm quark with $J^{P}=0^{-}$. Since it carries flavor explicitly and cannot annihilate into gluons, it is stable against the strong and electromagnetic annihilation processes and can only decay weakly, which provides a new window for studying the weak decay mechanism of heavy flavors. Another characteristic feature of the $B_{c}$ meson is that both of its constituent quarks

\footnotetext{
*liying@ytu.edu.cn
}

Published by the American Physical Society under the terms of the Creative Commons Attribution 4.0 International license. Further distribution of this work must maintain attribution to the author(s) and the published article's title, journal citation, and DOI. Funded by SCOAP ${ }^{3}$. are heavy and thus their weak decays give comparable contributions to the total decay rate. Therefore, the weak decay of the $B_{c}$ meson can be categorized into three classes: (i) the $b$-quark decays $(b \rightarrow c, u)$ with the $c$ quark as a spectator, which can be used to precisely determinate the Cabibbo-Kobayashi-Maskawa (CKM) matrix elements $\left|V_{c b}\right|$ and $\left|V_{u b}\right|$; (ii) the $c$-quark decays $(c \rightarrow s, d)$ with the $b$ quark as a spectator, which are suppressed by the phase space, but enhanced by the large CKM matrix elements $\left|V_{c s}\right|$ or $\left|V_{c d}\right|$; (iii) $b$-quark and $c$-quark coannihilation, which is enhanced by $\left|V_{c b} / V_{u b}\right|^{2} \sim 10^{2}$, in contrast to $B_{u}$ annihilation decays. The estimations of the $B_{c}$ decay rates indicate that the $c$-quark decays give the dominant contribution $(\sim 70 \%)$, while the $b$-quark decays and weak annihilation contribute about $\sim 20 \%$ and $\sim 10 \%$, respectively. All in all, the $B_{c}$ meson provides very rich weak decay channels to study perturbative and nonperturbative QCD dynamics and the annihilation mechanism of the $B$ meson, to test the standard model, as well as to search for signals of new physics [5]. In recent years, stimulated by both theoretical and experimental developments, many theoretical studies on the production and the semileptonic and nonleptonic decays of the $B_{c}$ meson have been explored by many groups based on the Isgur-Scora-Grinstein-Wise quark model [6], the relativistic independent quark model [7], QCD factorization $[8,9]$, the light-front quark model [9], SU(3) flavor symmetry [10], lattice gauge simulations [11], sum rules [12], nonrelativistic QCD methods [13], and the perturbative QCD (PQCD) approach [14-16]. 
In particular, in Refs. [14-16], the authors (including one of us) systematically investigated the $B_{c} \rightarrow D^{(\star)} P(V)$ decays (with $P$ and $V$ denoting the light pseudoscalar and vector meson) within the PQCD approach [17] based on the $k_{T}$ factorization. As known to all, the $B_{c}$ meson is a nonrelativistic heavy quarkonium system, and thus the two quarks in the $B_{c}$ meson are both at rest and nonrelativistic. Since the charm quark in the final-state $D$ meson is almost in the collinear state, a hard gluon is required to transfer large momentum to the spectator charm quark. So, the expansion based on $\alpha_{s}$ is reliable here. Moreover, we postulate a hierarchy $m_{B_{c}} \gg m_{D^{(*)}} \gg \Lambda_{\mathrm{QCD}}$. The relation $m_{B_{c}} \gg m_{D^{(*)}}$ justifies the perturbative analysis of the $B_{c} \rightarrow D^{(*)}$ form factors at large recoil and the definitions of light-cone $D^{(*)}$ meson wave functions. The relation $m_{D^{(*)}} \gg \Lambda_{\mathrm{QCD}}$ justifies the power expansion in the parameter $\Lambda_{\mathrm{QCD}} / m_{D^{(*)}}$. The small ratio $\Lambda_{\mathrm{QCD}} / m_{B_{c}}$ is viewed as being of higher power. So, the factorization theorem is applicable to the $B_{c}$ system similar to the situation of the $B$ meson with a light quark. Utilizing the $k_{T}$ factorization instead of collinear factorization, the PQCD approach does not have an end-point singularity, so the diagramsincluding factorizable, nonfactorizable, as well as annihilation-type diagrams - are all calculable. In Refs. [14-16], some branching fractions were found to be of the order of $\mathcal{O}\left(10^{-5}\right)$, which is measurable at the current LHCb experiment. For completeness, in this work we will extend previous studies to $B_{c} \rightarrow D^{(\star)} S$ decays where $S$ denotes a light scalar meson.

The light scalar mesons considered in this paper include the isosinglet $f_{0}(600)(\sigma), f_{0}(980), f_{0}(1370), f_{0}(1500) /$ $f_{0}(1710)$, the isodoublet $K_{0}^{*}(800)(\kappa)$ and $K_{0}^{*}(1430)$, and the isovector $a_{0}(980)$ and $a_{0}(1450)$ [18]. In the literature, the scalar mesons have been identified as ordinary $\bar{q} q$ states, four-quark states or meson-meson bound states, or even those supplemented with a scalar glueball; however, a definite conclusion has not been obtained. In light of the mass spectrum of scalar mesons and the strong and electromagnetic decays, most of us accept that the scalar mesons with masses below $1 \mathrm{GeV}$ constitute one nonet, while those near 1.5 GeV form another one [19]. Moreover, the scalar meson states above $1 \mathrm{GeV}$ can be identified as a conventional $q \bar{q}$ nonet with some possible glue content. However, the quark structure of the light scalar mesons below or near $1 \mathrm{GeV}$ has been quite controversial, though they are widely perceived as primarily the four-quark bound states. In the literature $[20,21]$, according to the category that the light mesons belong to, two typical scenarios for describing the scalar mesons have been proposed. Scenario 1 (S1) is the naive two-quark model: the nonet mesons below $1 \mathrm{GeV}$ [such as $\kappa, a_{0}(980), f_{0}(980)$, and $\sigma$ ] are treated as the lowestlying states, and accordingly those near $1.5 \mathrm{GeV}$ [such as $\left.a_{0}(1450), K_{0}(1430), f_{0}(1370 / 1500)\right]$ are the first orbitally excited states. In Scenario 2 (S2), the nonet mesons near
$1.5 \mathrm{GeV}$ are viewed as the lowest-lying states, while the mesons below $1 \mathrm{GeV}$ may be the exotic states beyond the quark model such as four-quark bound states. We have to stress that although experimental data indicates that the light scalar mesons [such as $f_{0}(980)$ and $a_{0}(980)$ ] are predominately four-quark states, in practice it is very hard for us to make quantitative predictions based on the four-quark picture because both the decay constants and the distribution amplitudes of $S$ are beyond the conventional quark model. Hence, we shall discuss only the two-quark scenario for light scalar mesons in the current work.

In the factorization hypothesis, for these considered $B_{c} \rightarrow D^{(*)} S$ decays with an emitted scalar meson, the factorizable emission amplitudes that are proportional to the matrix element $\langle S|(V \pm A)| 0\rangle$ will vanish or be tiny, because the neutral scalar mesons cannot be produced through the $(V \pm A)$ current and the decay constants of the charged scalar mesons are suppressed by the small difference between the two running current quark masses of the scalar meson. In order to obtain precise and reliable predictions, it is necessary for us to go beyond the naive factorization and calculate the contributions from the nonfactorizable diagrams, as well as the annihilation diagrams. We also note that for the considered $B_{c} \rightarrow D^{(*)} S$ decays, the annihilation-type diagrams will provide sizable contributions to the amplitudes and even dominate the amplitudes due to the enhancement from the large CKM matrix elements $V_{c b}$ and $V_{c s(d)}$. It is worth mentioning that the PQCD approach is an effective approach for calculating the nonfactorizable and annihilation diagrams, which can be confirmed by the precise predictions for the $B \rightarrow J / \psi D$ [22] and $B^{0} \rightarrow D_{s}^{-} K^{+}$decays [23]. So, for these considered decay channels, the predictions in the PQCD approach are reliable.

The remainder of the paper is organized as follows. The framework of PQCD, as well as the distribution amplitudes and decay constants of the mesons, are given in Sec. II. In Sec. III we present the formulas of each amplitude for each diagram. The numerical results and discussions are given in Sec. IV. We summarize this work in the last section.

\section{FRAMEWORK}

In this work, we shall describe the meson's momenta by using the light-cone coordinate. In the rest frame of the $B_{c}$ meson, the momenta of $B_{c}$, the scalar meson, and the $D$ meson, up to the order of $r_{D}^{2}$, are given by

$$
\begin{aligned}
P_{B_{c}} & =\frac{M_{B_{c}}}{\sqrt{2}}\left(1,1, \mathbf{0}_{\mathbf{T}}\right), \\
P_{2} & =\frac{M_{B_{c}}}{\sqrt{2}}\left(1-r_{D}^{2}, 0, \mathbf{0}_{\mathbf{T}}\right), \\
P_{3} & =\frac{M_{B_{c}}}{\sqrt{2}}\left(r_{D}^{2}, 1, \mathbf{0}_{\mathbf{T}}\right),
\end{aligned}
$$


where $r_{D}=m_{D} / m_{B_{c}}$. Note that the terms involving $r_{S}^{2}\left(r_{S}=m_{S} / m_{B_{c}}\right)$ are neglected in this work.

\section{A. PQCD approach}

It is known that in studying exclusive hadron decays the main theoretical uncertainties are from the calculations of matrix elements. The key point of the PQCD approach is to keep the intrinsic transverse momenta of the inner quarks, which is the so-called $k_{T}$ factorization [17]. The additional energy scale induced by the transverse momenta will lead to double logarithms in the QCD radiative corrections. Using the resummation technique, the double logarithms can be absorbed into the Sudakov form factor, which can suppress the long-distance contributions [24]. This Sudakov factor practically makes the PQCD approach applicable. Moreover, due to the radiative corrections of the weak vertex, another type of double logarithm $\alpha_{s} \ln ^{2} x$ (with $x$ being the momentum fraction of the inner quark) actually exists as $x \rightarrow 0$; therefore, these large corrections should also be resumed, which is called threshold resummation [25]. As a result, the end-point singularity in traditional collinear factorization can be smeared by this threshold factor.

There are several typical scales in the $B_{c}$ decays. In general, the factorization hypothesis is adopted to deal with processes with multiscales. As we already know, the physics higher than the scale of the $W$-boson mass $\left(m_{W}\right)$ can be calculated perturbatively, and the Wilson coefficients at the $m_{W}$ scale can be obtained. With the help of renormalization group techniques, we can get the Wilson coefficients from the $m_{W}$ scale to the $b$-quark mass $\left(m_{b}\right)$ scale. The hard part between the $m_{b}$ scale and the factorization scale $(t)$ can be calculated perturbatively in the PQCD approach. The physics lower than the $t$ scale belongs to the soft dynamics, which is nonperturbative but universal and can be parametrized into meson wave functions. The wave functions could be determined from experiments, or studied using the nonperturbative QCD approaches, such as QCD sum rules and lattice QCD. Therefore, in the PQCD approach the decay amplitude can be written as the convolution of the Wilson coefficients $C(t)$, the hard kernel $H\left(x_{i}, b_{i}, t\right)$, and the hadronic wave functions $\Phi_{B, D, S}\left(x_{i}, b_{i}\right)$ [26],

$$
\begin{aligned}
\mathcal{A} \sim & \int d x_{1} d x_{2} d x_{3} b_{1} d b_{1} b_{2} d b_{2} b_{3} d b_{3} \\
& \times \operatorname{Tr}\left[C(t) \Phi_{B}\left(x_{1}, b_{1}\right) \Phi_{S}\left(x_{2}, b_{2}\right) \Phi_{D}\left(x_{3}, b_{3}\right)\right. \\
& \left.\times H\left(x_{i},, b_{i}, t\right) S_{t}\left(x_{i}\right) e^{-S(t)}\right] .
\end{aligned}
$$

In Eq. (2), Tr denotes the trace over Dirac and color indices, the $x_{i}(i=1,2,3)$ are the momentum fractions of the "light" quark in each meson, and the $b_{i}$ are the conjugate variables of $k_{T i}$ of the valence quarks. $S_{t}\left(x_{i}\right)$ and $e^{-S(t)}$ are the threshold resummation and the Sudakov form factor, respectively.

\section{B. Wave functions of the $B_{c}$ and $D$ mesons}

In the PQCD approach, the universal nonperturbative wave functions are the most important inputs. Unlike $B_{u, d, s}$ mesons, our knowledge of the light-cone distribution amplitudes (LCDAs) for the $B_{c}$ meson is quite poor (for a recent view, see Ref. [27]). Although it has often been viewed as heavy quarkonium, we adopt the same form as the $B$ meson $[15,16]$,

$$
\Phi_{B_{c}}(x, b)=\frac{i}{\sqrt{6}}\left[\left(\not P+m_{B_{c}}\right) \gamma_{5} \phi_{B_{c}}(x, b)\right]
$$

Given $m_{B_{c}} \approx m_{b}+m_{c}$, the light-cone distribution amplitude $\phi_{B_{c}}(x, b)$ can be written as [27]

$$
\phi_{B_{c}}(x, b)=\frac{f_{B_{c}}}{2 \sqrt{6}} \delta\left(x-m_{c} / m_{B_{c}}\right) \exp \left[-\frac{1}{2} \omega^{2} b^{2}\right],
$$

where $m_{c}$ and $m_{b}$ are the mass of the charm quark and the beauty quark, respectively. $f_{B_{c}}$ is the decay constant of the $B_{c}$ meson. This simple form is the two-particle nonrelativistic LCDA at the leading order where both heavy valence quarks just share the total momentum of the $B_{c}$ mesons according to their masses. Since there is not enough experimental data to constrain the wave function and the distribution amplitude of the $B_{c}$ meson, the relativistic corrections and contributions from higher Fock states are not included in this work. The introduced factor $\exp \left[-\frac{1}{2} \omega^{2} b^{2}\right]$ represents the $k_{T}$ dependence in the PQCD approach, where $\omega=0.6 \pm 0.2$ is the shape parameter. In fact, the $B_{c}$ wave function for describing the intrinsic $k_{T}$ dependence has been studied for many years. Typically, the Gaussian form (also called the BrodskyHuang-Lepage form) was proposed [28] and applied to calculate the heavy meson decays [29]. Very recently, in Ref. [30] this form was reanalyzed and the parameters were fitted. Note that Eq. (4) is in agreement with the Gaussian form at leading power. We also acknowledge that there is a substantial value around the momentum fraction $x=m_{c} / m_{b} \sim 0.3$ within the width of about $\Lambda_{\mathrm{QCD}} / m_{b}$; however, it is the higher-power correction in light of the hierarchy $m_{B_{c}} \gg m_{D^{(*)}} \gg \Lambda_{\mathrm{QCD}}$, so we will not discuss this contribution and leave it to future work.

For the charmed $D^{(*)}$ mesons, following Ref. [31], we define the light-cone distribution amplitudes as

$$
\begin{aligned}
& \left\langle D(p)\left|q_{\alpha}(z) \bar{c}_{\beta}(0)\right| 0\right\rangle \\
& \quad=\frac{i}{2 \sqrt{6}} \int_{0}^{1} d x e^{i x p \cdot z}\left[\gamma_{5}\left(\not p+m_{D}\right) \phi_{D}(x, b)\right]_{\alpha, \beta}, \\
& \left\langle D^{*}(p)\left|q_{\alpha}(z) \bar{c}_{\beta}(0)\right| 0\right\rangle \\
& \quad=\frac{-1}{2 \sqrt{6}} \int_{0}^{1} d x e^{i x p \cdot z}\left[\phi_{L}\left(\not p+m_{D^{*}}\right) \phi_{D^{*}}^{L}(x, b)\right]_{\alpha, \beta},
\end{aligned}
$$


where the distribution amplitudes are

$$
\begin{aligned}
\phi_{D}(x, b)= & \phi_{D^{*}}^{L}(x, b) \\
= & \frac{1}{2 \sqrt{6}} f_{D^{(*)}} 6 x(1-x)\left[1+C_{D}(1-2 x)\right] \\
& \times \exp \left[-\frac{1}{2} \omega_{D}^{2} b^{2}\right],
\end{aligned}
$$

with $\omega_{D}=0.15 \pm 0.5 \mathrm{GeV}$. In this work, the high-twist distribution amplitudes are not included either, because

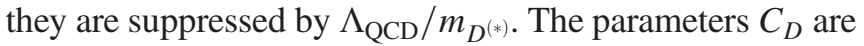
fitted from the $B \rightarrow D P(V)$ and $B_{s} \rightarrow D_{s} P(V)$ decays [31,32] and are set to be $C_{D}=0.5 \pm 0.1$ and $C_{D_{s}}=$ $0.4 \pm 0.1$, respectively.

\section{Physics of light scalar mesons}

Due to experimental developments, many scalar states have been discovered. Theoretically, as mentioned above, there are two different scenarios for describing the scalar mesons in the quark model. S1 is the typical two-quark model: the nonet mesons below $1 \mathrm{GeV}$ [including $f_{0}(600)(\sigma), f_{0}(980), K_{0}^{*}(800)(\kappa)$, and $\left.a_{0}(980)\right]$ belong to the lowest-lying states, and the ones near $1.5 \mathrm{GeV}$ [including $f_{0}(1370), f_{0}(1500) / f_{0}(1700), K_{0}^{*}(1430)$, and $\left.a_{0}(1450)\right]$ are viewed as the first excited states. In this scenario, the quark components of the light scalar mesons are given as

$$
\begin{array}{rlrl}
\sigma & =\frac{1}{\sqrt{2}}(u \bar{u}+d \bar{d}), \quad f_{0}=s \bar{s}, & \\
a_{0}^{+}=u \bar{d}, & a_{0}^{0}=\frac{1}{\sqrt{2}}(u \bar{u}+d \bar{d}), & a_{0}^{-}=d \bar{u}, \\
\kappa^{+}=u \bar{s}, \quad \kappa^{0}=d \bar{s}, \quad \bar{\kappa}^{0}=s \bar{d}, \quad \kappa^{-}=s \bar{u} .
\end{array}
$$

Here, $\sigma$ and $f_{0}(980)$ have an ideal mixing. In fact, the observed $D_{s} \rightarrow f_{0}(980) \pi^{+}$decay shows the probability of the $s \bar{s}$ component of $f_{0}(980)$, while $\Gamma\left(J / \psi \rightarrow f_{0}(980) \omega\right) \sim$ $\Gamma\left(J / \psi \rightarrow f_{0}(980) \phi\right)$ indicates the existence of the nonstrange components $[33,34]$. Based on the data, in the two-quark model $\sigma$ and $f_{0}(980)$ might be the mixing states as

$$
\begin{aligned}
\left|f_{0}(980)\right\rangle & =|s \bar{s}\rangle \cos \theta+|n \bar{n}\rangle \sin \theta, \\
|\sigma\rangle & =-|s \bar{s}\rangle \sin \theta+|n \bar{n}\rangle \cos \theta,
\end{aligned}
$$

with $|n \bar{n}\rangle=\frac{1}{\sqrt{2}}(u \bar{u}+d \bar{d})$ and $\theta$ is the mixing angle. As for the mixing angle $\theta$, we can determine it using various experimental measurements $[35,36]$. Currently, by analyzing the present experimental data, the two ranges $25^{\circ}<\theta<40^{\circ}$ and $140^{\circ}<\theta<165^{\circ}$ [37] are preferred. Similarly, $f_{0}(1370)$ and $f_{0}(1500)$ are the mixing states of $n \bar{n}, s \bar{s}$, and a glueball. In this paper, according to Ref. [38], we neglect the tiny contribution from the scalar glueball [39] and simplify the mixing form as

$$
\begin{aligned}
& f_{0}(1370)=0.78|n \bar{n}\rangle+0.51|s \bar{s}\rangle, \\
& f_{0}(1500)=-0.54|n \bar{n}\rangle+0.84|s \bar{s}\rangle .
\end{aligned}
$$

In $\mathrm{S} 2$, the nonet mesons near $1.5 \mathrm{GeV}$ are viewed as the lowest-lying states, while the mesons below $1 \mathrm{GeV}$ may be viewed as four-quark bound states. Because of the difficulty in dealing with four-quark states, we only do the calculation about the heavier nonet in S2.

Now, we shall discuss the decay constants and the distribution amplitudes of the scalar mesons. The two decay constants of scalar mesons are defined as

$$
\left\langle S(p)\left|\bar{q}_{2} \gamma_{\mu} q_{1}\right| 0\right\rangle=f_{S} p_{\mu}, \quad\left\langle S\left|\bar{q}_{2} q_{1}\right| 0\right\rangle=m_{S} \bar{f}_{S} .
$$

In terms of the charge-conjugation invariance, neutral scalar mesons cannot be produced by the vector current, so we obtain

$$
f_{\sigma}=f_{f_{0}}=f_{a_{0}^{0}}=0 .
$$

For other scalar mesons, the vector decay constant $f_{S}$ and the scalar one $\bar{f}_{S}$ are related by the equation of motion

$$
\bar{f}_{S}=\mu f_{S}, \quad \mu=\frac{m_{S}}{m_{2}(\mu)-m_{1}(\mu)},
$$

where $m_{S}$ is the mass of the scalar meson, and $m_{1}$ and $m_{2}$ are the running current quark masses. The inputs of the scalar mesons in our calculation-including the decay constants, the running quark masses in this paragraph, and the Gegenbauer moments in the following paragraphare quoted from Ref. [21].

In the two-quark model, the wave function of the scalar meson is given by

$$
\begin{aligned}
\left\langle S\left(P_{S}\right)\left|q(0)_{j} \bar{q}(z)_{l}\right| 0\right\rangle & \\
= & \frac{-1}{\sqrt{2 N_{c}}} \int_{0}^{1} d x e^{i x p \cdot z}\left\{\not p_{S} \phi_{S}(x)+m_{S} \phi_{S}^{s}(x)\right. \\
& \left.+m_{S}(\not h \not-1) \phi_{S}^{T}(x)\right\}_{j l},
\end{aligned}
$$

with the lightlike vectors $n=\left(1,0, \mathbf{0}_{T}\right)$ and $v=\left(0,1, \mathbf{0}_{T}\right)$. The twist-2 LCDA $\Phi_{S}(x)$ and twist-3 LCDAs $\phi_{S}^{s}(x)$ and $\phi_{S}^{\sigma}$ satisfy the normalization conditions

$$
\begin{aligned}
& \int_{0}^{1} d x \phi_{S}(x)=\frac{f_{S}}{2 \sqrt{2 N_{c}}} \\
& \int_{0}^{1} d x \phi_{S}^{s}(x)=\int_{0}^{1} d x \phi_{S}^{\sigma}(x)=\frac{\bar{f}_{S}}{2 \sqrt{2 N_{c}}} .
\end{aligned}
$$

The LCDAs can be expanded in Gegenbauer polynomials as follows:

$$
\phi_{S}(x)=\frac{f_{S}}{2 \sqrt{2 N_{c}}} 6 x(1-x)\left[1+\mu_{s} \sum_{m=1}^{\infty} B_{m}(\mu) C_{m}^{3 / 2}(2 x-1)\right],
$$




$$
\begin{aligned}
\phi_{S}^{s}(x)= & \frac{\bar{f}_{S}}{2 \sqrt{2 N_{c}}}\left[1+\sum_{m=1}^{\infty} a_{m}(\mu) C_{m}^{1 / 2}(2 x-1)\right], \\
\phi_{S}^{T}(x)= & \frac{d}{d x} \frac{\phi_{S}^{\sigma}(x)}{6}=\frac{\bar{f}_{S}}{2 \sqrt{2 N_{c}}} \frac{d}{d x} \\
& \times\left\{x(1-x)\left[1+\sum_{m=1}^{\infty} b_{m}(\mu) C_{m}^{3 / 2}(2 x-1)\right]\right\},
\end{aligned}
$$

where $B_{m}(\mu), a_{m}(\mu)$, and $b_{m}(\mu)$ are the Gegenbauer moments, and $C_{m}^{3 / 2}$ and $C_{m}^{1 / 2}$ are the Gegenbauer polynomials. The explicit values of $B_{m}(\mu)$ can be found in Ref. [21]. For the twist-3 LCDAs, we adopt the asymptotic form for simplicity, though the values of $b_{m}(\mu)$ and $a_{m}(\mu)$ have been explored [40].

\section{ANALYTIC FORMULAS}

For the considered decays, the weak effective Hamiltonian $\mathcal{H}_{\text {eff }}$ in the transition matrix elements can be written as [41]

$$
\begin{aligned}
\mathcal{H}_{\mathrm{eff}}= & \frac{G_{F}}{\sqrt{2}}\left[\sum _ { q = u , c } V _ { q b } ^ { * } V _ { q X } \left[C_{1}(\mu) O_{1}^{q}(\mu)\right.\right. \\
& \left.\left.+C_{2}(\mu) O_{2}^{q}(\mu)\right]-V_{t b}^{*} V_{t X} \sum_{i=3}^{10} C_{i}(\mu) O_{i}(\mu)\right],
\end{aligned}
$$

where $V_{q b(X)}$ and $V_{t b(X)}(X=d, s)$ are the CKM matrix elements, and $C_{i}(i=1-10)$ are the Wilson coefficients at the scale $\mu$. The $O_{i}(i=1, \ldots, 10)$ are the so-called local four-quark operators:

(1) Current-current (tree) operators,

$$
\begin{aligned}
& O_{1}^{q}=\left(\bar{b}_{\alpha} q_{\beta}\right)_{V-A}\left(\bar{q}_{\beta} X_{\alpha}\right)_{V-A}, \\
& O_{2}^{q}=\left(\bar{b}_{\alpha} q_{\alpha}\right)_{V-A}\left(\bar{q}_{\beta} X_{\beta}\right)_{V-A} .
\end{aligned}
$$

(2) QCD penguin operators,

$$
\begin{aligned}
& O_{3}=\left(\bar{b}_{\alpha} X_{\alpha}\right)_{V-A} \sum_{q^{\prime}}\left(\bar{q}_{\beta}^{\prime} q_{\beta}^{\prime}\right)_{V-A}, \\
& O_{4}=\left(\bar{b}_{\alpha} X_{\beta}\right)_{V-A} \sum_{q^{\prime}}\left(\bar{q}_{\beta}^{\prime} q_{\alpha}^{\prime}\right)_{V-A},
\end{aligned}
$$

$$
\begin{aligned}
& O_{5}=\left(\bar{b}_{\alpha} X_{\alpha}\right)_{V-A} \sum_{q^{\prime}}\left(\bar{q}_{\beta}^{\prime} q_{\beta}^{\prime}\right)_{V+A}, \\
& O_{6}=\left(\bar{b}_{\alpha} X_{\beta}\right)_{V-A} \sum_{q^{\prime}}\left(\bar{q}_{\beta}^{\prime} q_{\alpha}^{\prime}\right)_{V+A} .
\end{aligned}
$$

(3) Electroweak penguin operators

$$
\begin{gathered}
O_{7}=\frac{3}{2}\left(\bar{b}_{\alpha} X_{\alpha}\right)_{V-A} \sum_{q^{\prime}} e_{q^{\prime}}\left(\bar{q}_{\beta}^{\prime} q_{\beta}^{\prime}\right)_{V+A}, \\
O_{8}=\frac{3}{2}\left(\bar{b}_{\alpha} X_{\beta}\right)_{V-A} \sum_{q^{\prime}} e_{q^{\prime}}\left(\bar{q}_{\beta}^{\prime} q_{\alpha}^{\prime}\right)_{V+A}, \\
O_{9}=\frac{3}{2}\left(\bar{b}_{\alpha} X_{\alpha}\right)_{V-A} \sum_{q^{\prime}} e_{q^{\prime}}\left(\bar{q}_{\beta}^{\prime} q_{\beta}^{\prime}\right)_{V-A}, \\
O_{10}=\frac{3}{2}\left(\bar{b}_{\alpha} X_{\beta}\right)_{V-A} \sum_{q^{\prime}} e_{q^{\prime}}\left(\bar{q}_{\beta}^{\prime} q_{\alpha}^{\prime}\right)_{V-A} .
\end{gathered}
$$

Here $\alpha$ and $\beta$ are the color indices, and $q^{\prime}=(u, d, s, c, b)$ are the active quarks at the scale $m_{b} .(V-A)$ and $(V+A)$ are the left-handed and right-handed currents and are defined as $\left(\bar{b}_{\alpha} q_{\beta}\right)_{V-A}=\bar{b}_{\alpha} \gamma_{\mu}\left(1-\gamma_{5}\right) q_{\beta}$ and $\left(\bar{q}_{\beta}^{\prime} q_{\alpha}^{\prime}\right)_{V+A}=$ $\bar{q}_{\beta}^{\prime} \gamma_{\mu}\left(1+\gamma_{5}\right) q_{\alpha}^{\prime}$, respectively. The combined Wilson coefficients $a_{i}$ can be defined as [42]

$$
\begin{aligned}
a_{1} & =C_{2}+C_{1} / 3, & a_{2} & =C_{1}+C_{2} / 3, \\
a_{i} & =C_{i}+C_{i+1} / 3, & i & =3,5,7,9, \\
a_{j} & =C_{j}+C_{j-1} / 3, & j & =4,6,8,10 .
\end{aligned}
$$

According to the effective Hamiltonian, we can draw the possible lowest-order diagrams, as shown in Fig. 1, where the four diagrams in the first line are the emission diagrams and those in the second line are the annihilation diagrams. Now, we present the expressions for the hard kernels for all diagrams. After the perturbative calculation, when inserting different operators, the amplitudes for the factorizable emission diagrams in Figs. 1(a) and 1(b) are as follows.

(1) $(V-A)(V-A)$ :

$$
\begin{aligned}
\mathcal{A}_{e f}^{L L}= & 8 \pi C_{f} f_{S} m_{B_{c}}^{4} \int_{0}^{1} d x_{1} d x_{3} \int_{0}^{1 / \Lambda} b_{1} d b_{1} b_{3} d b_{3} \phi_{B_{c}}\left(x_{1}, b_{1}\right) \phi_{D}\left(x_{3}, b_{3}\right) \\
& \times\left\{\left[x_{3}\left(1-r_{D}^{2}\right)+r_{b}\left(r_{D}-2\right)-2 r_{D} x_{3}\right] E_{e f}\left(t_{a}\right) h_{a}+\left[\left(r_{D}-2\right) r_{D}\right] E_{e f}\left(t_{b}\right) h_{b}\right\} .
\end{aligned}
$$




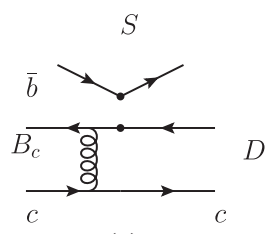

(a)

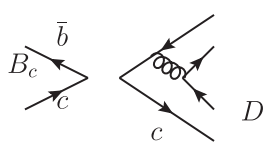

(e)

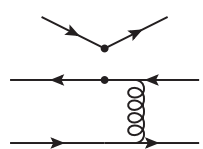

(b)

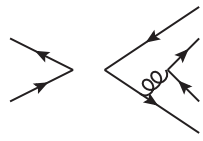

(f)

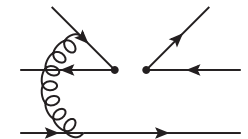

(c)

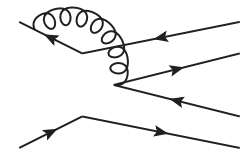

$(\mathrm{g})$

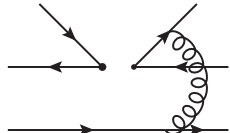

(d)

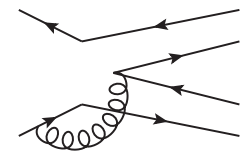

(h)

FIG. 1. Leading-order Feynman diagrams contributing to the $B_{c} \rightarrow D^{(*)} S$ decays in the PQCD approach. (a) and (b) are factorizable emission diagrams, (c) and (d) are the nonfactorizable emission ones; (e) and (f) are factorizable annihilation diagrams, (g) and (h) are the nonfactorizable emission ones.

(2) $(V-A)(V-A)$ :

$$
\mathcal{A}_{e f}^{L R}=\mathcal{A}_{\text {ef }}^{L L}
$$

(3) $(S-P)(S+P)$ :

$$
\begin{aligned}
\mathcal{A}_{e f}^{S P}= & 16 \pi C_{f} \bar{f}_{S} r_{S} m_{B_{c}}^{4} \int_{0}^{1} d x_{1} d x_{3} \int_{0}^{1 / \Lambda} b_{1} d b_{1} b_{3} d b_{3} \phi_{B_{c}}\left(x_{1}, b_{1}\right) \phi_{D}\left(x_{3}, b_{3}\right) \\
& \times\left\{\left[r_{b}+r_{D}\left(1+x_{3}\right)-2\right] E_{e f}\left(t_{a}\right) h_{a}+\left[2 r_{D}\left(x_{1}-1\right)-x_{1}\right] E_{e f}\left(t_{b}\right) h_{b}\right\} .
\end{aligned}
$$

In the above formulas, $r_{b}=m_{b} / m_{B_{c}}$ and $C_{f}=4 / 3$ is the group factor of $S U(3)_{c}$ for $B_{c}$ decays. The expressions for the scale $t$, Sudakov form factors $E$, and the hard functions $h$ can be found in the Appendix. Figures 1(c) and 1(d) are the nonfactorizable emission diagrams, whose contributions are as follows.

(1) $(V-A)(V-A)$ :

$$
\begin{aligned}
\mathcal{M}_{\text {enf }}^{L L}= & -16 \sqrt{\frac{2}{3}} \pi C_{f} m_{B_{c}}^{4} \int_{0}^{1} d x_{1} d x_{2} d x_{3} \int_{0}^{1 / \Lambda} b_{1} d b_{1} b_{2} d b_{2} \phi_{B_{c}}\left(x_{1}, b_{1}\right) \phi_{S}\left(x_{2}\right) \phi_{D}\left(x_{3}, b_{1}\right) \\
& \times\left\{\left[1-x_{1}-x_{2}+r_{D}\left(x_{3}-1\right)+r_{D}^{2}\left(x_{1}+2 x_{2}-x_{3}\right)\right] E_{\text {enf }}\left(t_{c}\right) h_{c}\right. \\
& \left.-\left[1-x_{1}+x_{2}-x_{3}+r_{D}\left(x_{3}-1\right)+r_{D}^{2}\left(x_{1}-2 x_{2}+x_{3}\right)\right] E_{\text {enf }}\left(t_{d}\right) h_{d}\right\} .
\end{aligned}
$$

(2) $(V-A)(V+A)$ :

$$
\begin{aligned}
& \mathcal{M}_{\text {enf }}^{L R}=16 \sqrt{\frac{2}{3}} \pi C_{f} m_{B_{c}}^{4} \int_{0}^{1} d x_{1} d x_{2} d x_{3} \int_{0}^{1 / \Lambda} b_{1} d b_{1} b_{2} d b_{2} \phi_{B_{c}}\left(x_{1}, b_{1}\right) \phi_{D}\left(x_{3}, b_{1}\right) \\
& \times\left\{\left[\phi_{S}^{S}\left(x_{2}\right)\left(x_{1}+x_{2}-1+r_{D}\left(x_{1}+x_{2}+x_{3}-2\right)\right)\right.\right. \\
& \left.+\phi_{S}^{T}\left(x_{2}\right)\left(x_{1}+x_{2}-1+r_{D}\left(x_{1}+x_{2}-x_{3}\right)\right)\right] \cdot E_{\text {enf }}\left(t_{c}\right) h_{c} \\
& -\left[\phi_{S}^{S}\left(x_{2}\right)\left(x_{1}-x_{2}+r_{D}\left(x_{1}-x_{2}+x_{3}-1\right)\right)\right. \\
& \left.\left.-\phi_{S}^{T}\left(x_{2}\right)\left(x_{1}-x_{2}+r_{D}\left(x_{1}-x_{2}-x_{3}+1\right)\right)\right] \cdot E_{\text {enf }}\left(t_{d}\right) h_{d}\right\} \text {. }
\end{aligned}
$$

(3) $(S-P)(S+P)$ :

$$
\begin{aligned}
\mathcal{M}_{\text {enf }}^{S P}= & -16 \sqrt{\frac{2}{3}} \pi C_{f} m_{B_{c}}^{4} \int_{0}^{1} d x_{1} d x_{2} d x_{3} \int_{0}^{1 / \Lambda} b_{1} d b_{1} b_{2} d b_{2} \phi_{B_{c}}\left(x_{1}, b_{1}\right) \phi_{S}\left(x_{2}\right) \phi_{D}\left(x_{3}, b_{1}\right) \\
& \times\left\{\left[2-x_{1}-x_{2}-x_{3}+r_{D}\left(x_{3}-1\right)+r_{D}^{2}\left(x_{1}+2 x_{2}+x_{3}-2\right)\right] \cdot E_{\text {enf }}\left(t_{c}\right) h_{c}\right. \\
& \left.-\left[-x_{1}+x_{2}+r_{D}\left(x_{3}-1\right)+r_{D}^{2}\left(x_{1}-2 x_{2}-x_{3}+2\right)\right] \cdot E_{\text {enf }}\left(t_{d}\right) h_{d}\right\} .
\end{aligned}
$$


As mentioned above, the annihilation-type diagrams can be calculated reliably in the PQCD approach. For the factorizable annihilation diagrams in Figs. 1(e) and 1(f), the expressions for hard kernels can be written as follows.

(1) $(V-A)(V-A)$ :

$$
\begin{aligned}
\mathcal{A}_{a f}^{L L}= & 8 \pi C_{f} f_{B_{c}} m_{B_{c}}^{4} \int_{0}^{1} d x_{2} d x_{3} \int_{0}^{1 / \Lambda} b_{2} d b_{2} b_{3} d b_{3} \phi_{D}\left(x_{3}, b_{3}\right) \\
& \times\left\{\left[\phi_{S}\left(x_{2}\right)\left(x_{3}-x_{3} r_{D}^{2}\right)+2 \phi_{S}^{S}\left(x_{2}\right) r_{S}\left(x_{3}+1\right) r_{D}\right] E_{a f}\left(t_{e}\right) h_{e}\right. \\
& -\left[\phi_{S}\left(x_{2}\right)\left(x_{2}-\left(1+2 x_{2}\right) r_{D}^{2}\right)+r_{S}\left(\phi_{S}^{S}\left(x_{2}\right) r_{D}\left(1+2 x_{2}\right)\right.\right. \\
& \left.\left.\left.-\phi_{S}^{T}\left(x_{2}\right) r_{D}\left(1-2 r_{D} x_{2}\right)\right)\right] E_{a f}\left(t_{f}\right) h_{f}\right\} .
\end{aligned}
$$

(2) $(V-A)(V+A)$ :

$$
\mathcal{A}_{a f}^{L R}=\mathcal{A}_{a f}^{L L}
$$

(3) $(S-P)(S+P)$ :

$$
\begin{aligned}
\mathcal{A}_{a f}^{S P}= & -16 \pi C_{f} f_{B_{c}} m_{B_{c}}^{4} \int_{0}^{1} d x_{2} d x_{3} \int_{0}^{1 / \Lambda} b_{2} d b_{2} b_{3} d b_{3} \phi_{D}\left(x_{3}, b_{3}\right) \\
& \times\left\{\left[\phi_{S}\left(x_{2}\right) r_{D} x_{3}+2 \phi_{S}^{S}\left(x_{2}\right) r_{S}\right] E_{a f}\left(t_{e}\right) h_{e}+\left[\phi_{S}\left(x_{2}\right) r_{D}+r_{S} x_{2}\left(\phi_{S}^{S}\left(x_{2}\right)-\phi_{S}^{T}\left(x_{2}\right)\right)\right] E_{a f}\left(t_{f}\right) h_{f}\right\} .
\end{aligned}
$$

For nonfactorizable annihilation diagrams in Figs. 1(g) and 1(h), the amplitudes are as follows.

(1) $(V-A)(V-A)$ :

$$
\begin{aligned}
& \mathcal{M}_{\text {anf }}^{L L}=-16 \sqrt{\frac{2}{3}} \pi C_{f} m_{B_{c}}^{4} \int_{0}^{1} d x_{1} d x_{2} d x_{3} \int_{0}^{1 / \Lambda} b_{1} d b_{1} b_{2} d b_{2} \phi_{B}\left(x_{1}, b_{1}\right) \phi_{D}\left(x_{3}, b_{2}\right) \\
& \times\left\{\left[\phi_{S}\left(x_{2}\right)\left(1-x_{1}-x_{2}-r_{b}+r_{D}^{2}\left(x_{1}+2 x_{2}-x_{3}\right)\right)-r_{D} r_{S}\left(\phi_{S}^{S}\left(x_{2}\right)\left(x_{1}+x_{2}+x_{3}-2\right)\right.\right.\right. \\
& \left.\left.+\phi_{S}^{T}\left(x_{2}\right)\left(x_{1}+x_{2}-x_{3}\right)\right)\right] E_{\text {anf }}\left(t_{g}\right) h_{g} \\
& +\left[\phi_{S}\left(x_{2}\right)\left(r_{D}+x_{3}\right)+r_{D} r_{S}\left(\phi_{S}^{S}\left(x_{2}\right)\left(x_{2}+x_{3}-x_{1}\right)\right.\right. \\
& \left.\left.\left.+\phi_{S}^{T}\left(x_{2}\right)\left(x_{1}-x_{2}+x_{3}\right)\right)\right] E_{\text {anf }}\left(t_{h}\right) h_{h}\right\} \text {. }
\end{aligned}
$$

(2) $(V-A)(V+A)$ :

$$
\begin{aligned}
\mathcal{M}_{\text {anf }}^{L R}= & -16 \sqrt{\frac{2}{3}} \pi C_{f} m_{B_{c}}^{4} \int_{0}^{1} d x_{1} d x_{2} d x_{3} \int_{0}^{1 / \Lambda} b_{1} d b_{1} b_{2} d b_{2} \phi_{B}\left(x_{1}, b_{1}\right) \phi_{D}\left(x_{3}, b_{2}\right) \\
& \times\left\{\left[\phi_{S}\left(x_{2}\right) r_{D}\left(x_{3}-2\right)-r_{S}\left(\phi_{S}^{S}\left(x_{2}\right)+\phi_{S}^{T}\left(x_{2}\right)\right)\left(x_{1}+x_{2}-2\right)\right] E_{\text {anf }}\left(t_{g}\right) h_{g}\right. \\
& \left.+\left[\phi_{S}\left(x_{2}\right) r_{D}\left(r_{D}-x_{3}\right)-r_{S}\left(\phi_{S}^{S}\left(x_{2}\right)+\phi_{S}^{T}\left(x_{2}\right)\right)\left(r_{D}+x_{1}-x_{2}\right)\right] E_{\text {anf }}\left(t_{h}\right) h_{h}\right\} .
\end{aligned}
$$

(3) $(S-P)(S+P)$ :

$$
\begin{aligned}
\mathcal{M}_{\text {anf }}^{S P}= & 16 \sqrt{\frac{2}{3}} \pi C_{f} m_{B_{c}}^{4} \int_{0}^{1} d x_{1} d x_{2} d x_{3} \int_{0}^{1 / \Lambda} b_{1} d b_{1} b_{2} d b_{2} \phi_{B}\left(x_{1}, b_{1}\right) \phi_{D}\left(x_{3}, b_{2}\right) \\
& \times\left\{\left[\phi_{S}\left(x_{2}\right)\left(1-r_{b}-x_{3}\right)-r_{D} r_{S}\left(\phi_{S}^{S}\left(x_{2}\right)\left(x_{1}+x_{2}+x_{3}-2\right)\right.\right.\right. \\
& \left.\left.-\phi_{S}^{T}\left(x_{2}\right)\left(x_{1}+x_{2}-x_{3}\right)\right)\right] E_{a n f}\left(t_{g}\right) h_{g} \\
& -\left[\phi_{S}\left(x_{2}\right)\left(x_{1}-x_{2}-r_{D}-r_{D}^{2}\left(x_{1}-2 x_{2}+x_{3}\right)\right)+r_{D} r_{S}\left(\phi_{S}^{S}\left(x_{2}\right)\left(x_{1}-x_{2}+x_{3}\right)\right.\right. \\
& \left.\left.\left.-\phi_{S}^{T}\left(x_{2}\right)\left(x_{2}+x_{3}-x_{1}\right)\right)\right] E_{a n f}\left(t_{h}\right) h_{h}\right\} .
\end{aligned}
$$


Similarly, the formulas for the $B_{c} \rightarrow D^{*} S$ decays are as follows:

$$
\begin{aligned}
& \mathcal{A}_{e f}^{* L L}=-8 \pi C_{f} f_{S} m_{B_{c}}^{4} \int_{0}^{1} d x_{1} d x_{3} \int_{0}^{1 / \Lambda} b_{1} d b_{1} b_{3} d b_{3} \phi_{B_{c}}\left(x_{1}, b_{1}\right) \phi_{D^{*}}^{L}\left(x_{3}, b_{3}\right) \\
& \times\left\{\left[r_{b}\left(r_{D}-2\right)-2 r_{D} x_{3}+x_{3}\left(1-r_{D}^{2}\right)\right] E_{e f}\left(t_{a}\right) h_{a}-r_{D}^{2} E_{e f}\left(t_{b}\right) h_{b}\right\}, \\
& \mathcal{A}_{e f}^{* L R}=\mathcal{A}_{e f}^{* L L} \\
& \mathcal{A}_{e f}^{* S P}=-16 \pi C_{f} \bar{f}_{S} r_{S} m_{B_{c}}^{4} \int_{0}^{1} d x_{1} d x_{3} \int_{0}^{1 / \Lambda} b_{1} d b_{1} b_{3} d b_{3} \phi_{B_{c}}\left(x_{1}, b_{1}\right) \phi_{D^{*}}^{L}\left(x_{3}, b_{3}\right) \\
& \times\left\{\left[2-r_{b}+r_{D}\left(1-x_{3}\right)\right] E_{e f}\left(t_{a}\right) h_{a}+x_{1} E_{e f}\left(t_{b}\right) h_{b}\right\}, \\
& \mathcal{M}_{\text {enf }}^{* L L}=16 \sqrt{\frac{2}{3}} \pi C_{f} m_{B_{c}}^{4} \int_{0}^{1} d x_{1} d x_{2} d x_{3} \int_{0}^{1 / \Lambda} b_{1} d b_{1} b_{2} d b_{2} \phi_{B_{c}}\left(x_{1}, b_{1}\right) \phi_{S}\left(x_{2}\right) \phi_{D^{*}}^{L}\left(x_{3}, b_{1}\right) \\
& \times\left\{\left[1-x_{1}-x_{2}+r_{D}\left(1-x_{3}\right)+r_{D}^{2}\left(x_{1}+2 x_{2}+x_{3}-2\right)\right] \cdot E_{\text {enf }}\left(t_{c}\right) h_{c}\right. \\
& \left.-\left[1-x_{1}+x_{2}-x_{3}+r_{D}\left(x_{3}-1\right)+r_{D}^{2}\left(x_{1}-2 x_{2}+x_{3}\right)\right] \cdot E_{\text {enf }}\left(t_{d}\right) h_{d}\right\} \text {, } \\
& \mathcal{M}_{\text {enf }}^{* L R}=-16 \sqrt{\frac{2}{3} \pi C_{f}} m_{B_{c}}^{4} \int_{0}^{1} d x_{1} d x_{2} d x_{3} \int_{0}^{1 / \Lambda} b_{1} d b_{1} b_{2} d b_{2} \phi_{B_{c}}\left(x_{1}, b_{1}\right) \phi_{D^{*}}^{L}\left(x_{3}, b_{1}\right) \\
& \times\left\{\left[\phi_{S}^{S}\left(x_{2}\right)\left(\left(r_{D}-1\right)\left(x_{1}+x_{2}\right)+r_{D} x_{3}+1\right)\right.\right. \\
& \left.+\phi_{S}^{T}\left(x_{2}\right)\left(1-x_{1}-x_{2}+r_{D}\left(x_{1}+x_{2}+x_{3}-2\right)\right)\right] \cdot E_{e n f}\left(t_{c}\right) h_{c} \\
& -\left[\phi_{S}^{S}\left(x_{2}\right)\left(x_{2}-x_{1}+r_{D}\left(x_{1}-x_{2}-x_{3}+1\right)\right)\right. \\
& \left.\left.-\phi_{S}^{T}\left(x_{2}\right)\left(x_{2}-x_{1}+r_{D}\left(x_{1}-x_{2}+x_{3}-1\right)\right)\right] \cdot E_{\text {enf }}\left(t_{d}\right) h_{d}\right\} \text {, } \\
& \mathcal{M}_{\text {enf }}^{* S P}=16 \sqrt{\frac{2}{3}} \pi C_{f} m_{B_{c}}^{4} \int_{0}^{1} d x_{1} d x_{2} d x_{3} \int_{0}^{1 / \Lambda} b_{1} d b_{1} b_{2} d b_{2} \phi_{B_{c}}\left(x_{1}, b_{1}\right) \phi_{S}\left(x_{2}\right) \phi_{D^{*}}^{L}\left(x_{3}, b_{1}\right) \\
& \times\left\{\left[r_{D}^{2}\left(x_{1}+2 x_{2}+x_{3}-2\right)+r_{D}\left(x_{3}-1\right)+2-x_{1}-x_{2}-x_{3}\right] E_{\text {enf }}\left(t_{c}\right) h_{c}\right. \\
& \left.-\left[r_{D}^{2}\left(x_{1}-2 x_{2}+x_{3}\right)+r_{D}\left(1-x_{3}\right)-x_{1}+x_{2}\right] E_{\text {enf }}\left(t_{d}\right) h_{d}\right\} \text {, } \\
& \mathcal{A}_{a f}^{* L L}=-8 \pi C_{f} f_{B_{c}} m_{B_{c}}^{4} \int_{0}^{1} d x_{2} d x_{3} \int_{0}^{1 / \Lambda} b_{2} d b_{2} b_{3} d b_{3} \phi_{D^{*}}^{L}\left(x_{3}, b_{3}\right) \\
& \times\left\{\left[\phi_{S}\left(x_{2}\right)\left(1-r_{D}^{2}\right) x_{3}+2 \phi_{S}^{S}\left(x_{2}\right) r_{S} r_{D}\left(x_{3}-1\right)\right] E_{a f}\left(t_{e}\right) h_{e}\right. \\
& \left.-\left[\phi_{S}\left(x_{2}\right)\left(r_{D}^{2}\left(1-2 x_{2}\right)+x_{2}\right)+r_{D} r_{s}\left(\phi_{S}^{S}\left(x_{2}\right)-\phi_{S}^{T}\left(x_{2}\right)\right)\right] E_{a f}\left(t_{f}\right) h_{f}\right\}, \\
& \mathcal{A}_{a f}^{* L R}=\mathcal{A}_{a f}^{* L L}, \\
& \mathcal{A}_{a f}^{* S P}=16 \pi C_{f} f_{B_{c}} m_{B_{c}}^{4} \int_{0}^{1} d x_{2} d x_{3} \int_{0}^{1 / \Lambda} b_{2} d b_{2} b_{3} d b_{3} \phi_{D^{*}}^{L}\left(x_{3}, b_{3}\right) \\
& \times\left\{\left[\phi_{S}\left(x_{2}\right) r_{D} x_{3}-2 \phi_{S}^{S}\left(x_{2}\right) r_{S}\right] E_{a f}\left(t_{e}\right) h_{e}\right. \\
& \left.-\left[\phi_{S}\left(x_{2}\right) r_{D}-r_{S} x_{2}\left(\phi_{S}^{T}\left(x_{2}\right)-\phi_{S}^{S}\left(x_{2}\right)\right)\right] E_{a f}\left(t_{f}\right) h_{f}\right\} \text {, }
\end{aligned}
$$




$$
\begin{aligned}
& \mathcal{M}_{\text {anf }}^{* L L}= 16 \sqrt{\frac{2}{3}} \pi C_{f} m_{B_{c}}^{4} \int_{0}^{1} d x_{1} d x_{2} d x_{3} \int_{0}^{1 / \Lambda} b_{1} d b_{1} b_{2} d b_{2} \phi_{B}\left(x_{1}, b_{1}\right) \phi_{D^{*}}^{L}\left(x_{3}, b_{2}\right) \\
& \times\left\{\left[\phi_{S}\left(x_{2}\right)\left(1-r_{b}-x_{1}-x_{2}+r_{D}^{2}\left(x_{1}+2 x_{2}+x_{3}-2\right)\right)-r_{D} r_{S}\left(\phi_{S}^{S}\left(x_{2}\right)\left(x_{1}+x_{2}-x_{3}\right)\right.\right.\right. \\
&+\left.\left.\phi_{S}^{T}\left(x_{2}\right)\left(x_{1}+x_{2}+x_{3}-2\right)\right)\right] \cdot E_{a n f}\left(t_{g}\right) h_{g} \\
&+\left[\phi_{S}\left(x_{2}\right)\left(r_{D}+\left(1-2 r_{D}^{2}\right) x_{3}\right)+r_{D} r_{S}\left(\phi_{S}^{S}\left(x_{2}\right)\left(x_{1}-x_{2}+x_{3}\right)\right.\right. \\
&\left.\left.+\phi_{S}^{T}\left(x_{2}\right)\left(x_{2}+x_{3}-x_{1}\right)\right] \cdot E_{a n f}\left(t_{h}\right) h_{h}\right\} \\
& \mathcal{M}_{\text {anf }}^{* L R}=-16 \sqrt{\frac{2}{3}} \pi C_{f} m_{B_{c}}^{4} \int_{0}^{1} d x_{1} d x_{2} d x_{3} \int_{0}^{1 / \Lambda} b_{1} d b_{1} b_{2} d b_{2} \phi_{B}\left(x_{1}, b_{1}\right) \phi_{D^{*}}^{L}\left(x_{3}, b_{2}\right) \\
& \times\left\{\left[\phi_{S}\left(x_{2}\right) r_{D}\left(x_{3}-2\right)-r_{S}\left(\phi_{S}^{S}\left(x_{2}\right)+\phi_{S}^{T}\left(x_{2}\right)\right)\left(x_{1}+x_{2}-2\right)\right] E_{a n f}\left(t_{g}\right) h_{g}\right. \\
&\left.+\left[\phi_{S}\left(x_{2}\right) r_{D}\left(r_{D}-x_{3}\right)-r_{S}\left(\phi_{S}^{S}\left(x_{2}\right)+\phi_{S}^{T}\left(x_{2}\right)\right)\left(r_{D}+x_{1}-x_{2}\right)\right] E_{a n f}\left(t_{h}\right) h_{h}\right\}, \\
& \mathcal{M}_{* a n f}^{S P}=-16 \sqrt{\frac{2}{3}} \pi C_{f} m_{B_{c}}^{4} \int_{0}^{1} d x_{1} d x_{2} d x_{3} \int_{0}^{1 / \Lambda} b_{1} d b_{1} b_{2} d b_{2} \phi_{B}\left(x_{1}, b_{1}\right) \phi_{D^{*}}^{L}\left(x_{3}, b_{2}\right) \\
& \times\left\{\left[\phi_{S}\left(x_{2}\right)\left(r_{b}+\left(1-2 r_{D}^{2}\right)\left(x_{3}-1\right)\right)\right.\right. \\
&\left.+r_{D} r_{S}\left(\phi_{S}^{T}\left(x_{2}\right)\left(x_{1}+x_{2}+x_{3}-2\right)-\phi_{S}^{S}\left(x_{2}\right)\left(x_{1}+x_{2}-x_{3}\right)\right)\right] E_{a n f}\left(t_{g}\right) h_{g} \\
&+\left[\phi_{S}\left(x_{2}\right)\left(x_{1}-x_{2}-r_{D}-r_{D}^{2}\left(x_{1}-2 x_{2}-x_{3}\right)\right)\right. \\
&\left.\left.+r_{D} r_{S}\left(\phi_{S}^{S}\left(x_{2}\right)\left(x_{1}-x_{2}+x_{3}\right)-\phi_{S}^{T}\left(x_{2}\right)\left(x_{1}-x_{2}-x_{3}\right)\right)\right] E_{a n f}\left(t_{h}\right) h_{h}\right\} .
\end{aligned}
$$

For the total decay amplitudes, the Wilson coefficients and the CKM elements are the same as for the corresponding $B_{c} \rightarrow D^{(*)} P$ decays with $P$ denoting a pseudoscalar meson (which can be found in Ref. [15]), since the topologies of these two types of decay are identical. As an example, we show the total decay amplitude of $B_{c} \rightarrow D^{+} K_{0}^{* 0}$ as

$$
\begin{aligned}
\mathcal{A}\left(B_{c}^{+} \rightarrow D^{+} K_{0}^{* 0}\right)= & \frac{G_{F}}{\sqrt{2}}\left\{V_{c b}^{*} V_{c s}\left(\mathcal{M}_{a f}^{L L} a_{1}+\mathcal{M}_{\text {anf }}^{L L} C_{1}\right)\right. \\
& -V_{t b}^{*} V_{t s}\left[\mathcal{M}_{\text {enf }}^{L L}\left(C_{3}-C_{9} / 2\right)+\mathcal{M}_{\text {enf }}^{L R}\left(C_{5}-C_{7} / 2\right)+\mathcal{M}_{a f}^{L L}\left(a_{4}+a_{10}\right)\right. \\
& \left.\left.+\mathcal{M}_{a f}^{S P}\left(a_{6}+a_{8}\right)+\mathcal{M}_{\text {anf }}^{L L}\left(C_{3}+C_{9}\right)+\mathcal{M}_{\text {anf }}^{L R}\left(C_{5}+C_{7}\right)\right]\right\} .
\end{aligned}
$$

The decay width of $B_{c} \rightarrow D^{(*)} S$ is given by

$$
\Gamma\left(B_{c} \rightarrow D^{(*)} S\right)=\frac{p}{8 \pi m_{B_{c}}^{2}}\left|\mathcal{A}\left(B_{c} \rightarrow D^{(*)} S\right)\right|^{2},
$$

where the momentum of the final-state particle is

$$
p=\frac{1}{2 m_{B_{c}}} \sqrt{\left[m_{B_{c}}^{2}-\left(m_{D}+m_{S}\right)^{2}\right]\left[m_{B_{c}}^{2}-\left(m_{D}-m_{S}\right)^{2}\right]} .
$$

\section{NUMERICAL RESULTS AND DISCUSSIONS}

In this section, we first list the other input parameters we used in the numerical calculations, such as the masses and lifetimes of mesons and the CKM matrix elements [18]:

$$
\begin{aligned}
\Lambda_{\mathrm{MS}}^{f=4} & =0.25 \pm 0.05 \mathrm{GeV}, \quad m_{B_{c}}=6.28 \mathrm{GeV}, \quad m_{b}=4.8 \mathrm{GeV}, \quad m_{D_{(s)}}=1.87 / 1.97 \mathrm{GeV}, \\
m_{D_{(s)}^{*}} & =2.01 / 2.11 \mathrm{GeV}, \quad \tau_{B_{c}}=0.46 p s, \quad \gamma=\left(69_{-11}^{+10}\right)^{\circ}, \quad \lambda=0.225 \pm 0.001, \quad V_{c b}=0.041_{-0.001}^{+0.001}, \\
V_{u s} & =0.225 \pm 0.001, \quad V_{t d}=0.009_{-0.001}^{+0.001}, V_{t s}=-0.040_{-0.001}^{+0.001} .
\end{aligned}
$$


TABLE I. The $C P$-averaged branching fractions (BFs) and $C P$ asymmetries of $B_{c} \rightarrow D S\left(a_{0}(980), \kappa, \sigma, f_{0}(980)\right)$ decays calculated in the PQCD approach in S1.

\begin{tabular}{|c|c|c|c|}
\hline Decay modes & Class & BFs $\left(10^{-6}\right)$ & $A_{C P}(\%)$ \\
\hline$B_{c} \rightarrow D^{+} a_{0}^{0}(980)$ & A & $3.44_{-0.75-1.69-0.32}^{+0.83+1.73+0.34}$ & $36.0_{-4.5-8.2-1.4}^{+5.0+7.0+0.5}$ \\
\hline$B_{c} \rightarrow D^{0} a_{0}^{+}(980)$ & A & $6.78_{-1.53-3.44-0.49}^{+1.71+3.33+0.52}$ & $1.88_{-2.09-3.93-0.79}^{+1.78+0.90+0.47}$ \\
\hline$B_{c} \rightarrow D^{+} \kappa_{0}^{0}(800)$ & A & $107_{-28-55-4}^{+32+65+3}$ & 0.0 \\
\hline$B_{c} \rightarrow D^{0} \kappa^{+}(800)$ & A & $93.6_{-24.7-50.7-3.0}^{+26.7+56.5+3.0}$ & $0.91_{-0.21-0.25-0.09}^{+0.23+0.30+0.06}$ \\
\hline$B_{c} \rightarrow D^{+} \sigma\left(f_{n}\right)$ & A & $3.39_{-0.71-1.30-0.10}^{+0.77+2.21+09}$ & $-36.4_{-3.8-5.3-3.2 .6}^{+3.6}$ \\
\hline$B_{c} \rightarrow D^{+} \sigma\left(f_{s}\right)$ & $\mathrm{P}$ & $0.02_{-0.01-0.01-0.00}^{+0.01+0.00}$ & 0.0 \\
\hline$B_{c} \rightarrow D^{+} f_{0}(980)\left(f_{n}\right)$ & A & $4.60_{-0.93-1.70-0.19+0.16}^{+1.04+2.19}$ & $-35.3_{-3.7-4.1-2.2}^{+3.3+6.2+2.6}$ \\
\hline$B_{c} \rightarrow D^{+} f_{0}(980)\left(f_{s}\right)$ & $\mathrm{P}$ & $0.02_{-0.01-0.01-0.00}^{+0.01+0.00}$ & 0.0 \\
\hline$B_{c} \rightarrow D_{s} a_{0}^{0}(980)$ & $\mathrm{C}$ & $0.03_{-0.01-0.01-0.00}^{+0.01+0.01+00}$ & $-2.28_{-0.26-1.15-0.12}^{+0.26+0.64+0.16}$ \\
\hline$B_{c} \rightarrow D_{s} \sigma\left(f_{n}\right)$ & $\mathrm{P}$ & $0.92_{-0.24-0.30-0.02}^{+0.27+0.03}$ & $1.12_{-0.26-0.26-0.12}^{+0.32+0.12}$ \\
\hline$B_{c} \rightarrow D_{s} \sigma\left(f_{s}\right)$ & A & $136_{-32-55-5}^{+32+94+4}$ & 0.0 \\
\hline$B_{c} \rightarrow D_{s} f_{0}(980)\left(f_{n}\right)$ & $P$ & $0.92_{-0.24-0.26-0.02}^{+0.27+0.49+0.03}$ & $1.12_{-0.26-0.26-0.12}^{+0.32+0.34+0.10}$ \\
\hline$B_{c} \rightarrow D_{s} f_{0}(980)\left(f_{s}\right)$ & A & $188_{-39-61-6}^{+42+69+6}$ & 0.0 \\
\hline$B_{c} \rightarrow D_{s} \bar{\kappa}_{0}^{0}(800)$ & A & $6.80_{-1.74-3.78-0.39}^{+1.70+4.45+0.40}$ & $-9.26_{-1.74-3.09-0.06}^{+1.65+2.10+0.06}$ \\
\hline
\end{tabular}

Within the above parameters, we calculated the $C P$ averaged branching fractions and the direct $C P$ asymmetries of all $40 B_{c} \rightarrow D^{(*)} S$ decays and we summarize the results in Tables I-IV. We acknowledge that there are many uncertainties in our work. In the tables, we mainly estimate three kinds of errors caused by the corresponding parameters. The first uncertainties come from the nonperturbative parameters, such as the decay constants and the distribution amplitudes. The second errors are from the high-order corrections. Since the next-to-leading-order corrections have not been determined, we vary the range of the hard scale $t$ as $(0.75 t \rightarrow 1.25 t)$ to estimate this kind of uncertainty. This strategy has been widely used in the studies of $B$ meson decays. The last errors arise from the uncertainties of the CKM matrix elements. Unlike the $B_{q}$ mesons, $B_{c}$ decays are dominated by the factorizable annihilations, the amplitudes of which are proportional to the decay constant of $B_{c}$, so the branching fractions are not sensitive to the distribution amplitude of the $B_{c}$ meson (less than 10\%) [16]. Therefore, we have not included the uncertainties taken by the $B_{c}$ wave function. We also emphasize that the next-to-leading power corrections will take large uncertainties; however, this kind of study is beyond the scope of the current paper, and we left it to future work. For convenience, in the tables we mark the dominant contributions of each decay mode by the symbols "C" (color-suppressed tree contributions), "A" ( $W$-annihilation-type contributions), and "P" (penguin contributions).

TABLE II. The $C P$-averaged branching fractions and the $C P$ asymmetries of $B_{c} \rightarrow D S\left(a_{0}(1450)\right.$, $\left.K_{0}^{*}(1430), f_{0}(1370), f_{0}(1500)\right)$ calculated in the PQCD approach in S1 and S2, respectively.

\begin{tabular}{|c|c|c|c|c|}
\hline Decay modes & Class & BFs $\left(10^{-6}\right)$ & $A_{C P}(\%)$ & Scenario \\
\hline \multirow[t]{2}{*}{$B_{c} \rightarrow D^{+} a_{0}^{0}(1450)$} & A & $5.30_{-1.72-1.20-0.03}^{+1.90+0.56+0.00}$ & $-27.7_{-10.6-3.6-3.5}^{+11.4+2.5+3.7}$ & $\mathrm{~S} 1$ \\
\hline & & $14.7_{-4.2-5.2-0.5}^{+4.7+4.7+0.8}$ & $13.6_{-4.1-2.4-2.0}^{+1.9+1.4+0.3}$ & $\mathrm{~S} 2$ \\
\hline \multirow[t]{2}{*}{$B_{c} \rightarrow D^{0} a_{0}^{+}(1450)$} & A & $9.89_{-3.26-2.84-0.32}^{+3.27+1.19+0.27}$ & $-7.89_{-4.62-1.52-0.92}^{+3.78+0.00+1.10}$ & $\mathrm{~S} 1$ \\
\hline & & $27.1_{-8.2-9.6-1.4}^{+8.9+9.9+1.5}$ & $1.91_{-1.12-2.86-0.42}^{+0.86+2.00+0.29}$ & $\mathrm{~S} 2$ \\
\hline \multirow[t]{2}{*}{$B_{c} \rightarrow D^{+} f_{0}(1370)$} & A & $1.22_{-0.37-0.38-0.17}^{+0.85+0.14+0.19}$ & $54.3_{-33.1-1.3-1.8}^{+30.1+27.3+0.4}$ & $\mathrm{~S} 1$ \\
\hline & & $7.01_{-2.06-2.67-0.46}^{+2.36+1.97+0.49}$ & $-20.4_{-3.9-4.7-0.6}^{+3.8+1.6+1.1}$ & $\mathrm{~S} 2$ \\
\hline \multirow[t]{2}{*}{$B_{c} \rightarrow D^{+} f_{0}(1500)$} & A & $0.94_{-0.30-0.10-0.09}^{+0.40+0.14+0.11}$ & $43.7_{-25.0-15.6-1.2}^{+28.1+19.0+0.4}$ & $\mathrm{~S} 1$ \\
\hline & & $3.60_{-1.11-1.24-0.11}^{+1.28+1.48+0.13}$ & $-22.8_{-5.4-3.0-0.9}^{+5.3+2.0+1.3}$ & $\mathrm{~S} 2$ \\
\hline \multirow[t]{2}{*}{$B_{c} \rightarrow D^{+} K_{0}^{* 0}(1430)$} & A & $191_{-43-49-6}^{+46+26+5}$ & 0.0 & $\mathrm{~S} 1$ \\
\hline & & $481_{-166-216-13}^{+175+170+14}$ & 0.0 & $\mathrm{~S} 2$ \\
\hline \multirow[t]{2}{*}{$B_{c} \rightarrow D^{0} K_{0}^{*+}(1430)$} & $\mathrm{A}$ & $193_{-40-40-6}^{+50+29+6}$ & $1.10_{-0.15-0.18-0.11}^{+0.17+0.45+0.09}$ & $\mathrm{~S} 1$ \\
\hline & & $458_{--166-176-13}^{+175+166+13}$ & $0.24_{-0.11-0.09-0.02}^{+0.12+0.06+0.02}$ & $\mathrm{~S} 2$ \\
\hline \multirow[t]{2}{*}{$B_{c} \rightarrow D_{s} a_{0}^{0}(1450)$} & $\mathrm{C}$ & $0.05_{-0.01-0.01-0.00}^{+0.02+0.02+0.00}$ & $-1.94_{-0.45-1.07-0.10}^{+0.28+1.11+0.15}$ & $\mathrm{~S} 1$ \\
\hline & & $0.02_{-0.01-0.01-0.00}^{+0.01+0.01+0.00}$ & $0.50_{-0.36-4.47-0.58}^{+0.80+0.86+0.03}$ & $\mathrm{~S} 2$ \\
\hline \multirow[t]{2}{*}{$B_{c} \rightarrow D_{s} f_{0}(1370)$} & A & $22.8_{-9.3-7.7-1.2}^{+16.7+2.3+1.1}$ & $-3.68_{-3.19-2.40-0.23}^{+2.53+0.16+0.30}$ & S1 \\
\hline & & $144_{-43-55-5}^{+46+42+5}$ & $1.12_{-0.21-0.10-0.10}^{+0.21+0.15+0.08}$ & $\mathrm{~S} 2$ \\
\hline \multirow[t]{2}{*}{$B_{c} \rightarrow D_{s} f_{0}(1500)$} & A & $113_{-46-36-3}^{+67+16+4}$ & $0.90_{-0.59-0.05-0.08}^{+0.83+0.51+0.07}$ & $\mathrm{~S} 1$ \\
\hline & & $209_{-62-24-9}^{+66+31+8}$ & $-0.66_{-0.09-0.06-0.05}^{+0.10+0.06+0.06}$ & $\mathrm{~S} 2$ \\
\hline \multirow[t]{2}{*}{$B_{c} \rightarrow D_{s} \bar{K}_{0}^{* 0}(1430)$} & A & $9.17_{-2.25-0.81-0.53}^{+2.40+1.81+0.55}$ & $7.18_{-096-1.91-0.05}^{+0.91+4.79+0.04}$ & $\mathrm{~S} 1$ \\
\hline & & $27.9_{-9.2-9.2-1.6}^{+10.8+13.0+1.7}$ & $-3.14_{-1.68-1.37-0.02}^{+1.06+1.36+0.02}$ & $\mathrm{~S} 2$ \\
\hline
\end{tabular}


TABLE III. The $C P$-averaged branching fractions and $C P$ asymmetries of $B_{c} \rightarrow D^{*} S\left(a_{0}, \kappa, \sigma, f_{0}\right)$ decays calculated in the PQCD approach in S1.

\begin{tabular}{|c|c|c|c|}
\hline Decay modes & Class & BFs $\left(10^{-6}\right)$ & $A_{C P}(\%)$ \\
\hline$\overline{B_{c} \rightarrow D^{*+} a_{0}^{0}(980)}$ & A & $3.38_{-0.59-1.34-0.20}^{+0.60+0.66+0.19}$ & $-69.8_{-6.0-7.9-3.5}^{+6.5+4.5+4.9}$ \\
\hline$B_{c} \rightarrow D^{* 0} a_{0}^{+}(980)$ & A & $5.42_{-0.89-2.11-0.29}^{+0.90+1.51+0.29}$ & $-28.4_{-3.6-4.3-1.7}^{+3.7+1.0+2.3}$ \\
\hline$B_{c} \rightarrow D^{*+} \sigma\left(f_{n}\right)$ & A & $1.65_{-0.25-0.59-0.06}^{+0.29+0.39+06}$ & $71.6_{-4.1-5.2-6.5}^{+3.8+5.6+5.5}$ \\
\hline$B_{c} \rightarrow D^{*+} \sigma\left(f_{s}\right)$ & $\mathrm{P}$ & $0.04_{-0.01-0.01-0.00}^{+0.01+0.01+00}$ & 0.0 \\
\hline$B_{c} \rightarrow D^{*+} f_{0}(980)\left(f_{n}\right)$ & A & $2.94_{-0.38-0.98-0.14}^{+0.40+0.57+0.13}$ & $58.7_{-4.1-3.2-4.8}^{+3.8+6.0+3.9}$ \\
\hline$B_{c} \rightarrow D^{*+} f_{0}(980)\left(f_{s}\right)$ & $\mathrm{P}$ & $0.04_{-0.01-0.01-0.00}^{+0.01+0.01+00}$ & 0.0 \\
\hline$B_{c} \rightarrow D^{*+} \kappa^{0}(800)$ & $\mathrm{A}$ & $54.8_{-9.1-23.3-1.6}^{+9.3+28.8+1.8}$ & 0.0 \\
\hline$B_{c} \rightarrow D^{* 0} \kappa^{+}(800)$ & A & $55.2_{-9.4-7.4-1.8}^{+10.6+21.6+1.8}$ & $0.10_{-0.25-0.17-0.01}^{+0.25+0.16+0.01}$ \\
\hline$B_{c} \rightarrow D_{s}^{*} a_{0}^{0}(980)$ & $\mathrm{C}$ & $0.06_{-0.01-0.01-0.00}^{+0.02+0.01+0.00}$ & $-2.84_{-0.12-1.05-0.15}^{+0.29+4.48+0.20}$ \\
\hline$B_{c} \rightarrow D_{s}^{*} \sigma\left(f_{n}\right)$ & $\mathrm{P}$ & $2.50_{-0.64-0.81-0.07}^{+0.72+1.68+0.08}$ & $1.65_{-0.14-0.57-0.18}^{+0.13+0.33+0.16}$ \\
\hline$B_{c} \rightarrow D_{s}^{*} \sigma\left(f_{s}\right)$ & A & $52.5_{-7.8-24.8-1.8}^{+7.8+8.8}$ & 0.0 \\
\hline$B_{c} \rightarrow D_{s}^{*} f_{0}(980)\left(f_{n}\right)$ & $\mathrm{P}$ & $2.50_{-0.64-0.81-0.07}^{+0.72+1.68+0.08}$ & $1.65_{-0.13-0.57-0.18}^{+0.13+0.33+0.16}$ \\
\hline$B_{c} \rightarrow D_{s}^{*} f_{0}(980)\left(f_{s}\right)$ & A & $108_{-14-50-4}^{+15+21+4}$ & 0.0 \\
\hline$B_{c} \rightarrow D_{s}^{*} \bar{\kappa}^{0}(800)$ & A & $3.07_{-0.47-0.97-0.18}^{+0.47+1.69+0.17}$ & $8.47_{-0.37-2.74-0.06}^{+0.51+2.63+0.05}$ \\
\hline
\end{tabular}

As we know, the quark components and physical properties of $f_{0}(980)$ and $\sigma$ are long-standing puzzles in particle physics. Although they are favored to be four-quark states, we here only assume $f_{0}(980)$ and $\sigma$ to be $n \bar{n}$ and $s \bar{s}$ bound states with a mixing, because in the four-quark scenario their wave functions and decay constants are still unknown. Besides many measurements of the charmless $B$ decays involving a scalar meson, the $\mathrm{LHCb}$ Collaboration also reported their first measurements of the charmed $B$ decays with a scalar $B\left(B_{s}\right) \rightarrow \bar{D} \sigma$ and $\bar{D} f_{0}(980)$ decays [43] at the end of 2015. Although we have large amounts of data, the mixing angle $\theta$ cannot be stringently constrained due to the large uncertainties [37]. In this work, using the two-quark model, for the sake of convenience we present the branching fractions of the $B_{c} \rightarrow D^{(*)} \sigma / f_{0}(980)$ decays individually under the pure $n \bar{n}$ and $s \bar{s}$ components. Having confirmed the two-quark model and fixed the mixing angle using other experiments, the branching fractions can be directly obtained from our predictions. As mentioned in Sec. II, we also present in Table $\mathrm{V}$ the branching fractions with mixing patterns by adopting two typical ranges$\left[25^{\circ}, 40^{\circ}\right]$ and $\left[140^{\circ}, 165^{\circ}\right]$ — where only the central values are quoted. As for $f_{0}(1370)$ and $f_{0}(1500)$, after neglecting the negligible glueball contents, the mixing form can be simplified as in Eq. (10). It is noted that in Tables II and IV, the presented branching fractions include the mixing patterns.

As stated in Ref. [3], the LHC experiment can produce about $10^{9} \quad B_{c}$ events every year. In Ref. [10], it was

TABLE IV. The $C P$-averaged branching fractions and $C P$ asymmetries of $B_{c} \rightarrow D^{*} S\left(a_{0}(1450)\right.$, $\left.K_{0}^{*}(1430), f_{0}(1370), f_{0}(1500)\right)$ calculated in the PQCD approach in S1 and S2, respectively.

\begin{tabular}{|c|c|c|c|c|}
\hline Decay modes & Class & BFs $\left(10^{-6}\right)$ & $A_{C P}(\%)$ & Scenario \\
\hline \multirow[t]{2}{*}{$B_{c} \rightarrow D^{*+} a_{0}^{0}(1450)$} & A & $2.18_{-0.59-0.90-0.48}^{+0.68+0.93+0.57}$ & $-45.5_{-19.9-7.2-5.8}^{+18.4+15.1+4.9}$ & $\mathrm{~S} 1$ \\
\hline & & $5.51_{-1.26-2.38-0.56}^{+1.42+2.81+0.62}$ & $-43.7_{-10.4-6.1-0.6}^{+9.8+4.2+0.6}$ & $\mathrm{~S} 2$ \\
\hline \multirow[t]{2}{*}{$B_{c} \rightarrow D^{* 0} a_{0}^{+}(1450)$} & A & $3.31_{-0.96-1.27-0.46}^{+0.89+1.62+0.53}$ & $-35.3_{-11.7-2.2-1.6}^{+14.3+22.1+1.9}$ & $\mathrm{~S} 1$ \\
\hline & & $10.7_{-2.3-4.1-0.8}^{+2.8+5.1+0.8}$ & $-16.5_{-4.6-3.5-0.4}^{+3.8+1.2+0.7}$ & $\mathrm{~S} 2$ \\
\hline \multirow[t]{2}{*}{$B_{c} \rightarrow D^{*+} f_{0}(1370)$} & $\mathrm{A}$ & $2.55_{-0.70-0.51-0.11}^{+0.90+0.54+0.07}$ & $0.12_{-5.58-2.32-0.20}^{+5.96+8.31+1.01}$ & $\mathrm{~S} 1$ \\
\hline & & $4.26_{-0.82-1.60-0.11}^{+1.00+1.76+0.08}$ & $24.2_{-6.6-1.5-2.6}^{+6.6+1.6+2.2}$ & $\mathrm{~S} 2$ \\
\hline \multirow[t]{2}{*}{$B_{c} \rightarrow D^{*+} f_{0}(1500)$} & A & $1.02_{-0.26-0.30-0.04}^{+0.27+0.42+0.02}$ & $-4.03_{-12.48-6.81-0.13}^{+14.78+14.00+0.12}$ & $\mathrm{~S} 1$ \\
\hline & & $2.35_{-0.47-1.08-0.07}^{+0.53+0.89+0.06}$ & $31.3_{-7.8-1.2-2.8}^{+8.0+2.5+2.3}$ & $\mathrm{~S} 2$ \\
\hline \multirow[t]{2}{*}{$B_{c} \rightarrow D^{*+} K_{0}^{* 0}(1430)$} & A & $63.3_{-13.8-22.6-2.0}^{+14.6+23.7+2.0}$ & 0.0 & $\mathrm{~S} 1$ \\
\hline & & $188_{-50-91-5}^{+51+93+4}$ & 0.0 & $\mathrm{~S} 2$ \\
\hline \multirow[t]{2}{*}{$B_{c} \rightarrow D^{* 0} K_{0}^{*+}(1430)$} & A & $61.7_{-12.6-13.3-1.6}^{+14.7+20.3+1.6}$ & $-0.25_{-0.41-0.20-0.01}^{+0.41+0.33+0.02}$ & $\mathrm{~S} 1$ \\
\hline & & $192_{-46-83-4}^{+51+90+5}$ & $-0.07_{-0.21-0.11-0.01}^{+0.21+0.13+0.01}$ & $\mathrm{~S} 2$ \\
\hline \multirow[t]{2}{*}{$B_{c} \rightarrow D_{s}^{*} a_{0}^{0}(1450)$} & $\mathrm{C}$ & $0.14_{-0.05-0.04-0.01}^{+0.04+0.05+0.01}$ & $-1.44_{-0.47-1.23-0.08}^{+0.32+1.92+0.11}$ & $\mathrm{~S} 1$ \\
\hline & & $0.05_{-0.02-0.01-0.00}^{+0.02+0.01+00}$ & $-0.15_{-0.87-1.06-0.01}^{+0.56+55+0.01}$ & $\mathrm{~S} 2$ \\
\hline \multirow[t]{2}{*}{$B_{c} \rightarrow D_{s}^{*} f_{0}(1370)$} & A & $34.2_{-9.2-10.8-0.1}^{+10.6+12.2+0.1}$ & $-0.89_{-0.45-0.38-0.17}^{+0.55+0.20+0.16}$ & S1 \\
\hline & & $72.4_{-14.9-30.7-1.3}^{+17.1+35.1+1.3}$ & $-2.09_{-0.52-0.32-0.18}^{+0.49+0.18+0.22}$ & $\mathrm{~S} 2$ \\
\hline \multirow[t]{2}{*}{$B_{c} \rightarrow D_{s}^{*} f_{0}(1500)$} & A & $28.5_{-6.8-7.9-0.8}^{+8.8+8.6+0.7}$ & $0.95_{-0.80-0.11-0.15}^{+0.70+0.29+0.16}$ & $\mathrm{~S} 1$ \\
\hline & & $186_{-42-82-5}^{+55+94+5}$ & $1.01_{-0.25-0.10-0.04}^{+0.29+0.18+0.03}$ & $\mathrm{~S} 2$ \\
\hline \multirow[t]{2}{*}{$B_{c} \rightarrow D_{s}^{*} \bar{K}_{0}^{* 0}(1430)$} & $\mathrm{A}$ & $4.87_{-0.97-1.34-0.28}^{+1.15+1.90+0.30}$ & $-4.89_{-1.03-1.05-0.02}^{+0.80+0.68+0.03}$ & $\mathrm{~S} 1$ \\
\hline & & $11.3_{-2.7-5.2-0.7}^{+2.9+6.3+0.6}$ & $4.22_{-2.10-1.91-0.02}^{+1.09+0.16+0.02}$ & $\mathrm{~S} 2$ \\
\hline
\end{tabular}


TABLE V. The calculated branching fractions of $B_{c} \rightarrow D^{(*)} f_{0}(980)$ and $\sigma$ with the mixing in the PQCD approach (in units of $10^{-6}$ ).

\begin{tabular}{|c|c|c|c|c|}
\hline \multirow[b]{2}{*}{ Decay modes } & \multicolumn{2}{|c|}{$\left[25^{\circ}, 40^{\circ}\right]$} & \multicolumn{2}{|c|}{$\left[140^{\circ}, 165^{\circ}\right]$} \\
\hline & $\mathrm{BF}\left(10^{-6}\right)$ & $A_{C P}(\%)$ & $\mathrm{BF}\left(10^{-6}\right)$ & $A_{C P}(\%)$ \\
\hline$B_{c} \rightarrow D^{+} \sigma$ & $2.82 \sim 2.04$ & $-29.6 \sim-26.3$ & $1.95 \sim 3.14$ & $-41.1 \sim-36.1$ \\
\hline$B_{c} \rightarrow D^{+} f_{0}(980)$ & $0.94 \sim 2.04$ & $-23.1 \sim-26.7$ & $1.77 \sim 0.25$ & $-37.3 \sim-49.9$ \\
\hline$B_{c} \rightarrow D_{s} \sigma$ & $19.9 \sim 50.3$ & $-3.84 \sim-1.96$ & $63.9 \sim 13.4$ & $1.57 \sim 3.82$ \\
\hline$B_{c} \rightarrow D_{s} f_{0}(980)$ & $160 \sim 117$ & $0.60 \sim 1.06$ & $104 \sim 172$ & $-1.19 \sim-0.37$ \\
\hline$B_{c} \rightarrow D^{*+} \sigma$ & $1.29 \sim 0.90$ & $76.6 \sim 80.2$ & $1.05 \sim 1.57$ & $62.0 \sim 68.6$ \\
\hline$B_{c} \rightarrow D^{*+} f_{0}(980)$ & $0.57 \sim 1.25$ & $38.2 \sim 47.6$ & $1.21 \sim 0.22$ & $67.7 \sim 76.4$ \\
\hline$B_{c} \rightarrow D_{s}^{*} \sigma$ & $10.5 \sim 22.0$ & $8.98 \sim 5.44$ & $24.3 \sim 6.44$ & $-4.72 \sim-8.65$ \\
\hline$B_{c} \rightarrow D_{s}^{*} f_{0}(980)$ & $89.8 \sim 65.1$ & $-1.47 \sim-2.59$ & $64.0 \sim 101$ & $2.68 \sim 0.86$ \\
\hline
\end{tabular}

estimated that the charmless $B_{c}$ decays with a branching fraction at the level $10^{-6}$ yield a few events per year at LHCb. Because the selection criteria and the trigger efficiencies are very different for each decay mode, in order to roughly estimate the expected sensitivity for the considered $B_{c} \rightarrow D S$ decays it is necessary to base the quantitative analysis on the numerical results. Based on our predictions, we believe that some $B_{c} \rightarrow D S$ decays with large decay rates will be detected in the experiments, such as LHCb and CMS. Taking the decay $B_{c}^{+} \rightarrow D^{+} K_{0}^{* 0}(1430)$ as an example, the branching fraction is predicted to be about $1.9 \times 10^{-4}$ in $\mathrm{S} 1$. On the experimental side, we in particular use the charged final states to reconstruct $D^{+}$ and $K_{0}^{* 0}(1430)$, the branching fractions of which are $\mathcal{B}\left(D^{+} \rightarrow K^{-} \pi^{+} \pi^{-}\right) \simeq 10 \%$ and $\mathcal{B}\left(K_{0}^{* 0} \rightarrow K^{+} \pi^{-}\right) \simeq 45 \%$ [18]. According to Ref. [44], if the total efficiency is assumed to be $1 \%$, about 200 events per year can be expected at the $\mathrm{LHCb}$ experiment. Since the branching fraction of $B_{c}^{+} \rightarrow D^{+} K_{0}^{* 0}(1430)$ in S2 is a bit larger than in $\mathrm{S} 1$, we can expect more events to be detected. As for $B_{c}^{+} \rightarrow D^{*+} K_{0}^{* 0}(1430)$ involving a vector charmed meson, the situation is similar, as the vector $D^{*}$ meson decays to a $D$ meson with a rate close to $100 \%$. Based on our predictions, the decays with branching fractions in the range $\left[10^{-5}, 10^{-4}\right]$ are expected to be measured in the near future.

On the basis of the numerical results we obtained, we provide the following discussion.

(1) For the decays with an emitted scalar, the contribution $\mathcal{A}_{\text {ef }}^{(*) L}$ will vanish or be suppressed, because the neutral scalar meson cannot be produced through the local $(V \pm A)$ current, or the vector decay constants of the charged scalar mesons are highly suppressed by the tiny mass difference between the two running current quark masses. Because the factorizable emission diagrams are forbidden, the $B_{c}^{+} \rightarrow D_{s}^{(*)} a_{0}^{0}(980 / 1450)$ decays are only induced by the nonfactorizable emission diagrams $(\mathrm{C})$; therefore, these modes have tiny branching fractions. Generally, in contrast to the emission contributions, the annihilation-type contributions are power suppressed in the charmless $B_{u, d, s}$ decays. However, in $B_{c}$ decays the annihilation-type contributions play major roles because of the large enhancement by the Wilson coefficient $a_{1}$ and the CKM matrix elements $V_{c s(d)}$. In fact, this situation is similar to the $B_{c} \rightarrow D^{(*)} T$ decays in Ref. [16] with $T$ denoting a tensor meson. For this reason, most considered decays are dominated by the $W$-annihilation-type contributions (A), as classified in the tables. In particular, the $B_{c}^{+} \rightarrow D_{(s)}^{(*)+} \sigma / f_{0}(980)$ and $B_{c}^{+} \rightarrow$ $D_{(s)}^{(*)+} f_{0}(1370 / 1500)$ decays are also dominated by the annihilations, though the final scalars are a mix between $n \bar{n}$ and $s \bar{s}$.

(2) Inevitably, there are large theoretical uncertainties in the numerical calculations; in particular, the properties of the scalar meson are not well understood, and the wave function of the $B_{c}$ meson is not very accurate. In order to reduce the dependence of the input parameters, we thus define two ratios as

$$
\begin{gathered}
\frac{\mathcal{B}\left(B_{c}^{+} \rightarrow D^{(*) 0} a_{0}^{+}\right)}{\mathcal{B}\left(B_{c}^{+} \rightarrow D^{(*)+} a_{0}^{0}\right)} \sim 2, \\
\frac{\mathcal{B}\left(B_{c}^{+} \rightarrow D^{(*)+} K_{0}^{* 0}\right)}{\mathcal{B}\left(B_{c}^{+} \rightarrow D^{(*) 0} K_{0}^{*+}\right)} \sim 1 .
\end{gathered}
$$

In Ref. [15], it was found that $\mathcal{B}\left(B_{c}^{+} \rightarrow D^{(*) 0} \pi^{+} / \rho^{+}\right) \gg$ $\mathcal{B}\left(B_{c}^{+} \rightarrow D^{(*)+} \pi^{0} / \rho^{0}\right)$, where the $B_{c}^{+} \rightarrow D^{(*)} \pi^{+} / \rho^{+}$ modes are dominated by the factorizable emission diagrams, while the color-suppressed modes $B_{c}^{+} \rightarrow$ $D^{(*)+} \pi^{0} / \rho^{0}$ are dominated by the annihilation diagrams. The relation $\mathcal{B}\left(B_{c}^{+} \rightarrow D^{(*) 0} \pi^{+} / \rho^{+}\right) \gg$ $\mathcal{B}\left(B_{c}^{+} \rightarrow D^{(*)+} \pi^{0} / \rho^{0}\right)$ means that in $B_{c} \rightarrow D P(V)$ the annihilation-type contributions are suppressed, compared with the contributions of the factorizable emission diagrams. However, when the scalar is involved, because both $B_{c}^{+} \rightarrow D^{(*) 0} a_{0}^{+}$and $B_{c}^{+} \rightarrow$ $D^{(*)+} a_{0}^{0}$ are dominated by the annihilation-type 
contribution, the relation $\mathcal{B}\left(B_{c}^{+} \rightarrow D^{(*) 0} a_{0}^{+}\right) \sim$ $2 \mathcal{B}\left(B_{c}^{+} \rightarrow D^{(*)+} a_{0}^{0}\right)$ is understandable. Similarly, we can also explain the relation $\mathcal{B}\left(B_{c}^{+} \rightarrow D^{(*)+} K_{0}^{* 0}\right) \approx$ $\mathcal{B}\left(B_{c}^{+} \rightarrow D^{(*) 0} K_{0}^{*+}\right)$.

(3) The $B_{c}^{+} \rightarrow D^{0} K^{+}$and $B_{c}^{+} \rightarrow D^{+} K^{0}$ decays are affected by the penguin operators in addition to the annihilations [15]. Compared with the contribution from annihilations, the penguin emission diagrams have a sizable contribution but with a relative minus sign. So, their branching fractions are much smaller than those of the corresponding $B_{c}^{+} \rightarrow$ $D^{0} \kappa^{+} / K_{0}^{*+}(1430)$ and $B_{c}^{+} \rightarrow D^{+} \kappa^{0} / K_{0}^{* 0}(1430)$ decays with the large annihilation contribution alone, because the emission contributions are highly suppressed. We also note that the magnitudes of $B_{c}^{+} \rightarrow$ $D^{(*) 0} \kappa / K_{0}^{*}(1430)$ are $\mathcal{O}\left(10^{-4}\right)$, which is measurable at LHCb. The measurement of such decays will afford a few hints for studying the annihilation mechanisms in $B$ physics.

(4) As expected, the branching fractions of the four "C"-type decays are much smaller than the "A"-type decays, and the contributions of the penguin operators are negligible. Although the four decays also have a large Wilson coefficient $\left(C_{2} \sim 1.0\right)$, they are suppressed by the tiny CKM factors. For example, the CKM factor of $B_{c}^{+} \rightarrow D_{s}^{+} a_{0}$ is $V_{u b}^{*} V_{u s}$, and that of $B_{c}^{+} \rightarrow D^{+} a_{0}$ is $V_{u b}^{*} V_{u d}$.

(5) From Tables II and IV, we find that for the "A"-type decays the branching fractions in $\mathrm{S} 2$ are about 2-3 times larger than those in $\mathrm{S} 1$, except for $B_{c}^{+} \rightarrow D_{(s)}^{(*)+} f_{0}(1370) / f_{0}(1500)$. However, for the "C"'-type $B_{c}^{+} \rightarrow D_{s}^{(*)} a_{0}^{0}(1450)$ decays, the branching fractions in S2 are much smaller than those in $\mathrm{S} 1$, which illustrates that the contribution of the annihilation-type diagrams in S2 is much larger than in S1. Somewhat differently, the contribution of hard-scattering emission diagrams in $\mathrm{S} 2$ is much smaller than that in S1. This phenomena is due to the different signs of the decay constants under different scenarios.

(6) We now discuss the decay modes involving $f_{0}(1370,1500)$ that are mixed states of $n \bar{n}$ and $s \bar{s}$. In $\mathrm{S} 1$, the inference between the $n \bar{n}$ component and the $s \bar{s}$ component is destructive for $B_{c}^{+} \rightarrow$ $D^{+} f_{0}(1370)$, while it is constructive for $B_{c}^{+} \rightarrow$ $D^{+} f_{0}(1500)$. Therefore, the branching fraction of $B_{c}^{+} \rightarrow D^{+} f_{0}(1500)$ is about the same as $B_{c}^{+} \rightarrow$ $D^{+} f_{0}(1370)$, although $B_{c}^{+} \rightarrow D^{+} f_{0}(1500)$ is suppressed by the mixing coefficient with respect to $B_{c}^{+} \rightarrow D^{+} f_{0}(1370)$. For the $B_{c}^{+} \rightarrow D^{*+} f_{0}(1370) /$ $f_{0}(1500)$ decays, the opposite applies. The inference is constructive (destructive) for $B_{c}^{+} \rightarrow D^{+} f_{0}(1500)$ $\left[B_{c}^{+} \rightarrow D^{+} f_{0}(1370)\right]$, because the wave functions of $D$ and $D^{*}$ have different signs, as shown in Eqs. (5) and (6). As a result, the branching fraction of $B_{c}^{+} \rightarrow$ $D^{*+} f_{0}(1500)$ is much smaller than that of $B_{c}^{+} \rightarrow$ $D^{*+} f_{0}(1370)$. Similarly, the interference can also explain the relations $\mathcal{B}\left(B_{c}^{+} \rightarrow D_{s} f_{0}(1370) \times\right.$ $\left.\left(S_{1}\right)\right) \ll \mathcal{B}\left(B_{c}^{+} \rightarrow D_{s} f_{0}(1500)\left(S_{1}\right)\right)$ and $\mathcal{B}\left(B_{c}^{+} \rightarrow\right.$ $\left.D_{s}^{*} f_{0}(1370)\left(S_{1}\right)\right) \sim \mathcal{B}\left(B_{c}^{+} \rightarrow D_{s}^{*} f_{0}(1500)\left(S_{1}\right)\right)$. In $\mathrm{S} 2$, because the contributions from $s \bar{s}$ ("C" type) are negligible, the interference between $n \bar{n}$ and $s \bar{s}$ in $B_{c}^{+} \rightarrow D^{(*)+} f_{0}(1370) / f_{0}(1500)$ decays is weak, and thus we obtain

$$
\frac{\mathcal{B}\left(B_{c}^{+} \rightarrow D^{+} f_{0}(1370)\right)}{\mathcal{B}\left(B_{c}^{+} \rightarrow D^{+} f_{0}(1500)\right)} \sim \frac{\mathcal{B}\left(B_{c}^{+} \rightarrow D^{*+} f_{0}(1370)\right)}{\mathcal{B}\left(B_{c}^{+} \rightarrow D^{*+} f_{0}(1500)\right)} \sim 2 .
$$

Similarly, we have

$\frac{\mathcal{B}\left(B_{c}^{+} \rightarrow D_{s} f_{0}(1500)\right)}{\mathcal{B}\left(B_{c}^{+} \rightarrow D_{s} f_{0}(1370)\right)} \sim \frac{\mathcal{B}\left(B_{c}^{+} \rightarrow D_{s}^{*} f_{0}(1500)\right)}{\mathcal{B}\left(B_{c}^{+} \rightarrow D_{s}^{*} f_{0}(1370)\right)} \sim \frac{3}{2}$.

(7) From the tables, it is apparent that the direct $C P$ asymmetries are very small, since the contributions from penguin operators are much smaller than those from the tree operators, except for the $B_{c}^{+} \rightarrow$ $D^{(*)+} a_{0}^{0}(980 / 1450)$ and $B_{c}^{+} \rightarrow D^{(*)+} \sigma / f_{0}(980)$ decays. For $B_{c}^{+} \rightarrow D^{(*)+} a_{0}^{0}(980 / 1450)$, the contribution from tree operators is suppressed by the cancellation between the nonfactorizable emission diagrams and the annihilation-type diagrams, so that the interference between the contributions from the tree operators and those from the penguin operators are sizable and the direct $C P$ asymmetries become large. For the $B_{c}^{+} \rightarrow D^{(*)+} \sigma / f_{0}(980)$ decays, the contributions from the $f_{s}$ component are small, because the factorizable emission diagrams are forbidden and the nonfactorizable contributions are suppressed by the CKM matrix elements. This is why these decays are dominated by the $f_{n}$ component. For the $f_{n}$ component, the contribution from penguin operators is comparable to that from tree operators, because the latter contribution becomes small due to the cancellation between emission and annihilation diagrams. So the $B_{c}^{+} \rightarrow$ $D^{(*)+} \sigma / f_{0}(980)$ decays have large direct $C P$ asymmetries in the two-quark picture. Unfortunately, these $C P$ asymmetries cannot be measured at the current $\mathrm{LHCb}$ experiment, as their branching fractions are too small. We also note that the $C P$ asymmetries of the $B_{c}^{+} \rightarrow D^{*+} f 0(1370) / f_{0}(1500)$ decays are heavily dependent on the scenarios, which might be useful for identifying different 
scenarios when the experiments are available in the near future.

\section{CONCLUSION}

In this work, within the PQCD approach, we studied the branching fractions and $C P$ asymmetries of $40 B_{c} \rightarrow D S$ decays involving scalar mesons. As it is the only heavy meson consisting of two heavy quarks with different flavors, the $B_{c}$ meson's wave function is not well defined, and thus here we adopted the $\delta$ function. Because the quark components of the scalars have not been confirmed, two different scenarios have been discussed. It is worth noting that the nonperturbative parameters and the corrections from higher orders and higher powers are beyond the scope of this work and not included in this work, which can be left for future work. After the calculation, we found that several branching fractions are in the range $\left[10^{-5}, 10^{-4}\right]$, some of which could be measured at the LHCb experiment, and other decays with smaller fractions might be measured at other high-energy colliders. Furthermore, we also note that some decays have large $C P$ asymmetries, but they are unmeasurable currently due to small branching fractions.

\section{ACKNOWLEDGMENTS}

This work was supported in part by the National Science Foundation of China under the Grant Nos. 11575151, $11705159,11235005,11765012,11447032$, by the Natural Science Foundation of Shandong province (ZR2014AQ013 and ZR2016JL001), and by the Research Fund of Jiangsu Normal University under Grant No. HB2016004. Y. L. is grateful to the Institute of High Energy Physics (IHEP) for hospitality where this work was initiated.

\section{APPENDIX: RELATED HARD FUNCTIONS}

In this appendix, we summarize the functions that appear in the analytic formulas in Sec. III. First, we present the auxiliary functions as

$$
\begin{aligned}
& F_{0}^{2}=m_{B_{c}}^{2}\left(x_{1}-r_{D}^{2}\right)\left(1-x_{3}\right), \\
& F_{a}^{2}=m_{B_{c}}^{2}\left(r_{b}^{2}-x_{3}\left(1-r_{D}^{2}\right)\right),
\end{aligned}
$$

$$
F_{b}^{2}=m_{B_{c}}^{2}\left(x_{1}-r_{D}^{2}\right)
$$

$$
\begin{gathered}
F_{c}^{2}=m_{B_{c}}^{2}\left(x_{3}-1\right)\left(\left(1-r_{D}^{2}\right)\left(1-x_{2}\right)-\left(x_{1}-r_{D}^{2}\right)\right), \\
F_{d}^{2}=m_{B_{c}}^{2}\left(x_{3}-1\right)\left(\left(1-r_{D}^{2}\right) x_{2}-\left(x_{1}-r_{D}^{2}\right)\right), \\
E_{0}^{2}=m_{B_{c}}^{2}\left(x_{2} x_{3}\left(1-r_{D}^{2}\right)\right), \\
F_{e}^{2}=m_{B_{c}}^{2}\left(x_{3}\left(1-r_{D}^{2}\right)\right), \\
F_{f}^{2}=m_{B_{c}}^{2}\left(\left(\left(1-r_{D}^{2}\right) x_{2}+r_{D}^{2}\right)-r_{c}^{2}\right), \\
F_{g}^{2}=m_{B_{c}}^{2}\left(r_{b}^{2}-\left(1-x_{3}\right)\left(1-x_{1}-x_{2}\left(1-r_{D}^{2}\right)\right)\right), \\
F_{h}^{2}=m_{B_{c}}^{2}\left(r_{c}^{2}+x_{3}\left(x_{1}-x_{2}\left(1-r_{D}^{2}\right)\right)\right) .
\end{gathered}
$$

The hard scales $t_{i}$ can be determined by

$$
\begin{aligned}
& t_{a}=\max \left\{\sqrt{\left|F_{0}^{2}\right|}, \sqrt{\left|F_{a}^{2}\right|}, 1 / b_{1}, 1 / b_{3}\right\}, \\
& t_{b}=\max \left\{\sqrt{\left|F_{0}^{2}\right|}, \sqrt{\left|F_{b}^{2}\right|}, 1 / b_{1}, 1 / b_{3}\right\}, \\
& t_{c}=\max \left\{\sqrt{\left|F_{0}^{2}\right|}, \sqrt{\left|F_{c}^{2}\right|}, 1 / b_{1}, 1 / b_{2}\right\}, \\
& t_{d}=\max \left\{\sqrt{\left|F_{0}^{2}\right|}, \sqrt{\left|F_{d}^{2}\right|}, 1 / b_{1}, 1 / b_{2}\right\}, \\
& t_{e}=\max \left\{\sqrt{\left|E_{0}^{2}\right|}, \sqrt{\left|F_{e}^{2}\right|}, 1 / b_{2}, 1 / b_{3}\right\}, \\
& t_{f}=\max \left\{\sqrt{\left|E_{0}^{2}\right|}, \sqrt{\left|F_{f}^{2}\right|}, 1 / b_{2}, 1 / b_{3}\right\}, \\
& t_{h}=\max \left\{\sqrt{\left|E_{0}^{2}\right|}, \sqrt{\left|F_{g}^{2}\right|}, 1 / b_{1}, 1 / b_{2}\right\}, \\
& \left.t_{\left|E_{0}^{2}\right|}, \sqrt{\left|F_{h}^{2}\right|}, 1 / b_{1}, 1 / b_{2}\right\} .
\end{aligned}
$$

The hard functions are written as

$$
\begin{aligned}
& h_{a}=K_{0}\left(\sqrt{\left|F_{0}^{2}\right|} b_{1}\right)\left\{\begin{array}{l}
\theta\left(b_{1}-b_{3}\right) I_{0}\left(\sqrt{\left|F_{a}^{2}\right|} b_{3}\right) K_{0}\left(\sqrt{\left|F_{a}^{2}\right|} b_{1}\right)+\theta\left(b_{3}-b_{1}\right) I_{0}\left(m_{B_{c}} \sqrt{\left|F_{a}^{2}\right|} b_{1}\right) K_{0}\left(\sqrt{\left|F_{a}^{2}\right|} b_{3}\right) \quad F_{a}^{2}>0, \\
{\left[\theta\left(b_{1}-b_{3}\right) J_{0}\left(\sqrt{\left|F_{a}^{2}\right|} b_{3}\right) H_{0}^{(1)}\left(\sqrt{\left|F_{a}^{2}\right|} b_{1}\right)+\theta\left(b_{3}-b_{1}\right) J_{0}\left(\sqrt{\left|F_{a}^{2}\right|} b_{1}\right) H_{0}^{(1)}\left(\sqrt{\left|F_{a}^{2}\right|} b_{3}\right)\right] F_{a}^{2}<0,}
\end{array}\right. \\
& h_{b}=K_{0}\left(\sqrt{\left|F_{0}^{2}\right|} b_{3}\right)\left\{\begin{array}{l}
\left.\theta\left(b_{1}-b_{3}\right) I_{0}\left(\sqrt{\left|F_{b}^{2}\right|} b_{3}\right) K_{0} \sqrt{\left|F_{b}^{2}\right|} b_{1}\right)+\theta\left(b_{3}-b_{1}\right) I_{0}\left(\sqrt{\left|F_{b}^{2}\right|} b_{1}\right) K_{0}\left(\sqrt{\left|F_{b}^{2}\right|} b_{3}\right) \\
{\left[\theta\left(b_{1}-b_{3}\right) J_{0}\left(\sqrt{\left|F_{b}^{2}\right|} b_{3}\right) H_{0}^{(1)}\left(\sqrt{\left|F_{b}^{2}\right|} b_{1}\right)+\theta\left(b_{3}-b_{1}\right) J_{0}\left(\sqrt{\left|F_{b}^{2}\right|} b_{1}\right) H_{0}^{(1)}\left(\sqrt{\left|F_{b}^{2}\right|} b_{3}\right)\right] F_{b}^{2}<0,}
\end{array}\right.
\end{aligned}
$$




$$
h_{c}=\left[\theta\left(b_{2}-b_{1}\right) K_{0}\left(\sqrt{\left|F_{0}^{2}\right|} b_{2}\right) I_{0}\left(\sqrt{\left|F_{0}^{2}\right|} b_{1}\right)+\theta\left(b_{1}-b_{2}\right) K_{0}\left(\sqrt{\left|F_{0}^{2}\right|} b_{1}\right) I_{0}\left(\sqrt{\left|F_{0}^{2}\right|} b_{2}\right)\right] \cdot \begin{cases}\frac{i \pi}{2} H_{0}^{(1)}\left(\sqrt{\left|F_{c}^{2}\right|} b_{2}\right), & F_{c}^{2}<0, \\ K_{0}\left(\sqrt{\left|F_{c}^{2}\right|} b_{2}\right), & F_{c}^{2}>0,\end{cases}
$$

$h_{d}=\left[\theta\left(b_{2}-b_{1}\right) K_{0}\left(\sqrt{\left|F_{0}^{2}\right|} b_{2}\right) I_{0}\left(\sqrt{\left|F_{0}^{2}\right|} b_{1}\right)+\theta\left(b_{1}-b_{2}\right) K_{0}\left(\sqrt{\left|F_{0}^{2}\right|} b_{1}\right) I_{0}\left(\sqrt{\left|F_{0}^{2}\right|} b_{2}\right)\right] \cdot \begin{cases}\frac{i \pi}{2} H_{0}^{(1)}\left(\sqrt{\left|F_{d}^{2}\right|} b_{2}\right), & F_{d}^{2}<0 \\ K_{0}\left(\sqrt{\left|F_{d}^{2}\right|} b_{2}\right), & F_{d}^{2}>0\end{cases}$

$$
h_{e}=\left(\frac{i \pi}{2}\right)^{2} H_{0}^{(1)}\left(\sqrt{\left|E_{0}^{2}\right|} b_{2}\right)\left[\theta\left(b_{2}-b_{3}\right) H_{0}^{(1)}\left(\sqrt{\left|F_{e}^{2}\right|} b_{2}\right) J_{0}\left(\sqrt{\left|F_{e}^{2}\right|} b_{3}\right)+\theta\left(b_{3}-b_{2}\right) H_{0}^{(1)}\left(\sqrt{\left|F_{e}^{2}\right|} b_{3}\right) J_{0}\left(\sqrt{\left|F_{e}^{2}\right|} b_{2}\right)\right] \cdot S_{t}\left(x_{3}\right) \text {, }
$$

$h_{f}=\left(\frac{i \pi}{2}\right)^{2} H_{0}^{(1)}\left(\sqrt{\left|E_{0}^{2}\right|} b_{2}\right)\left[\theta\left(b_{2}-b_{3}\right) H_{0}^{(1)}\left(\sqrt{\left|F_{f}^{2}\right|} b_{2}\right) J_{0}\left(\sqrt{\left|F_{f}^{2}\right|} b_{3}\right)+\theta\left(b_{3}-b_{2}\right) H_{0}^{(1)}\left(\sqrt{\left|F_{f}^{2}\right|} b_{3}\right) J_{0}\left(\sqrt{\left|F_{f}^{2}\right|} b_{2}\right)\right] \cdot S_{t}\left(x_{3}\right)$,

$h_{g}=\frac{i \pi}{2}\left[\theta\left(b_{1}-b_{2}\right) H_{0}^{(1)}\left(\sqrt{\left|E_{0}^{2}\right|} b_{1}\right) J_{0}\left(\sqrt{\left|E_{0}^{2}\right|} b_{2}\right)+\theta\left(b_{2}-b_{1}\right) H_{0}^{(1)}\left(\sqrt{\left|E_{0}^{2}\right|} b_{2}\right) J_{0}\left(\sqrt{\left|E_{0}^{2}\right|} b_{1}\right)\right] \times \begin{cases}\frac{i \pi}{2} H_{0}^{(1)}\left(\sqrt{\left|F_{g}^{2}\right|} b_{1}\right), & F_{g}^{2}<0, \\ K_{0}\left(\sqrt{\left|F_{g}^{2}\right|} b_{1}\right), & F_{g}^{2}>0,\end{cases}$

$$
h_{h}=\frac{i \pi}{2}\left[\theta\left(b_{1}-b_{2}\right) H_{0}^{(1)}\left(\sqrt{\left|E_{0}^{2}\right|} b_{1}\right) J_{0}\left(\sqrt{\left|E_{0}^{2}\right|} b_{2}\right)+\theta\left(b_{2}-b_{1}\right) H_{0}^{(1)}\left(\sqrt{\left|E_{0}^{2}\right|} b_{2}\right) J_{0}\left(\sqrt{\left|E_{0}^{2}\right|} b_{1}\right)\right] \times \begin{cases}\frac{i \pi}{2} H_{0}^{(1)}\left(\sqrt{\left|F_{h}^{2}\right|} b_{1}\right), F_{h}^{2}<0, \\ K_{0}\left(\sqrt{\left|F_{h}^{2}\right|} b_{1}\right), & F_{h}^{2}>0 .\end{cases}
$$

$S_{t}(x)$ is the jet function from the threshold resummation, which can be written as [25]

$$
S_{t}(x)=\frac{2^{1+2 c} \Gamma(3 / 2+c)}{\sqrt{\pi} \Gamma(1+c)}[x(1-x)]^{c},
$$

with $c=0.3$. The evolution functions $E_{i}$ and $E_{\text {enf }}\left(t_{b}\right)$ in the analytic formulas are given by

$$
\begin{array}{r}
E_{e f}(t)=\alpha_{s}(t) \exp \left[-S_{B_{c}}(t)-S_{D}(t)\right], \\
E_{\text {enf }}(t)=\left.\alpha_{s}(t) \exp \left[-S_{B_{c}}(t)-S_{D}(t)-S_{S}(t)\right]\right|_{b_{1}=b_{3}}, \\
E_{a f}(t)=\alpha_{s}(t) \exp \left[-S_{D}(t)-S_{S}(t)\right] \mid, \\
E_{\text {anf }}(t)=\left.\alpha_{s}(t) \exp \left[-S_{B_{c}}(t)-S_{D}(t)-S_{S}(t)\right]\right|_{b_{2}=b_{3}} .
\end{array}
$$

The Sudakov exponents are defined as

$$
S_{B_{c}}(t)=s\left(x_{1} \frac{m_{B_{c}}}{\sqrt{2}}, b_{1}\right)+\frac{5}{3} \int_{1 / b_{1}}^{t} \frac{d \bar{\mu}}{\bar{\mu}} \gamma_{q}\left(\alpha_{s}(\bar{\mu})\right),
$$

$$
S_{D}(t)=s\left(x_{3} \frac{m_{B_{c}}}{\sqrt{2}}, b_{3}\right)+2 \int_{1 / b_{3}}^{t} \frac{d \bar{\mu}}{\bar{\mu}} \gamma_{q}\left(\alpha_{s}(\bar{\mu})\right)
$$

$$
\begin{aligned}
S_{S}(t)= & s\left(x_{2}\left(1-r_{D}^{2}\right) \frac{m_{B_{c}}}{\sqrt{2}}, b_{2}\right) \\
& +s\left(\left(1-x_{2}\right)\left(1-r_{D}^{2}\right) \frac{m_{B_{c}}}{\sqrt{2}}, b_{3}\right) \\
& +2 \int_{1 / b_{2}}^{t} \frac{d \bar{\mu}}{\bar{\mu}} \gamma_{q}\left(\alpha_{s}(\bar{\mu})\right),
\end{aligned}
$$

where $s(Q, b)$ can be found in Ref. [17]. 
[1] F. Abe et al. (CDF Collaboration), Phys. Rev. D 58, 112004 (1998); Phys. Rev. Lett. 81, 2432 (1998).

[2] A. Abulencia et al. (CDF Collaboration), Phys. Rev. Lett. 97, 012002 (2006); T. Aaltonen et al. (CDF Collaboration), Phys. Rev. Lett. 100, 182002 (2008); V. M. Abazov et al. (D0 Collaboration), Phys. Rev. Lett. 101, 012001 (2008).

[3] J. He (LHCb collaboration), in Proceedings of the 17th International Workshop on Deep-Inelastic Scattering and Related Subjects (DIS 2009), Madrid, Spain, edited by C. Glasman and J. Terron (Science Wise, Berlin, 2009).

[4] R. Aaij et al. (LHCb Collaboration), Phys. Rev. Lett. 108, 251802 (2012); Phys. Rev. Lett. 111, 181801 (2013); G. Aad et al. (ATLAS Collaboration), Phys. Rev. Lett. 113, 212004 (2014).

[5] N. Brambilla et al. (Quarkonium Working Group Collaboration), Report No. CERN-2005-005; N. Brambilla et al., Eur. Phys. J. C 71, 1534 (2011).

[6] N. Sharma, R. Dhir, and R. C. Verma, J. Phys. G 37, 075013 (2010); N. Sharma, Phys. Rev. D 81, 014027 (2010); N. Sharma and R. C. Verma, Phys. Rev. D 82, 094014 (2010); G. L. Castro, H. B. Mayorga, and J. H. Muñoz, J. Phys. G 28, 2241 (2002).

[7] S. Naimuddin, S. Kar, M. Priyadarsini, N. Barik, and P. C. Dash, Phys. Rev. D 86, 094028 (2012); N. Barik, S. Naimuddin, P. C. Dash, and S. Kar, Phys. Rev. D 80, 074005 (2009).

[8] J. F. Sun, G. F. Xue, Y. L. Yang, G. R. Lu, and D. S. Du, Phys. Rev. D 77, 074013 (2008).

[9] H.-M. Choi and C.-R. Ji, Phys. Rev. D 80, 114003 (2009).

[10] S. Descotes-Gemon, J. He, E. Kou, and P. Robbe, Phys. Rev. D 80, 114031 (2009).

[11] C. T. H. Davies, K. Hornbostel, G. P. Lepage, A. J. Lidsey, J. Shigemitsu, and J. Sloan, Phys. Lett. B 382, 131 (1996); I. F. Allison, C. T. H. Davies, A. Gray, A. S. Kronfeld, P. B. Mackenzie, and J. N. Simone, Nucl. Phys. B, Proc. Suppl. 140, 440 (2005); H. P. Shanahan, P. Boyle, C. T. H. Davies, and H. Newton, Phys. Lett. B 453, 289 (1999); B. D. Jones and R. M. Woloshyn, Phys. Rev. D 60, 014502 (1999).

[12] V. V. Kiselev, J. Phys. G 30, 1445 (2004); V. V. Kiselev, A. E. Kovalsky, and A. K. Likhoded, arXiv:hep-ph/ 0006104; V. V. Kiselew, A. E. Kovalsky, and A. K. Likhoded, Nucl. Phys. B585, 353 (2000); V. V. Kiselev, arXiv:hep-ph/0211021.

[13] C. H. Chang and Y. Q. Chen, Phys. Rev. D 49, 3399 (1994); C. H. Chang, Y. Q. Chen, and R. J. Oakes, Phys. Rev. D 54, 4344 (1996); M. Lusignoli, M. Masetti, and S. Perarca, Phys. Lett. B 266, 142 (1991); N. Brambilla and A. Vairo, Phys. Rev. D 62, 094019 (2000); N. Brambilla, A. Pineda, J. soto, and A. Vairo, Rev. Mod. Phys. 77, 1423 (2005).

[14] J. F. Cheng, D. S. Du, and C. D. Lu, Eur. Phys. J. C 45, 711 (2006); X. Liu, Z. J. Xiao, and C. D. Lu, Phys. Rev. D 81, 014022 (2010); X. Liu and Z. J. Xiao, Phys. Rev. D 82, 054029 (2010); 81, 074017 (2010); J. Phys. G 38, 035009 (2011); Z. J. Xiao and X. Liu, Phys. Rev. D 84, 074033 (2011); Chin. Sci. Bull. 59, 3748 (2014).

[15] Z. Rui, Z. T. Zou, and C. D. Lu, Phys. Rev. D 86, 074008 (2012).

[16] Z. T. Zou, X. Yu, and C. D. Lu, Phys. Rev. D 87, 074027 (2013).
[17] Y. Y. keum, H. N. Li, and A. I. Sanda, Phys. Lett. B 504, 6 (2001); Phys. Rev. D 63, 054008 (2001).C. D. Lu, K. Ukai, and M. Z. Yang, Phys. Rev. D 63, 074009 (2001).H. N. Li, Prog. Part. Nucl. Phys. 51, 85 (2003) and references therein.

[18] C. Patrignani et al. (Particle Data Group Collaboration), Chin. Phys. C 40, 100001 (2016) and 2017 update.

[19] F. E. Close and N. A. Törnqvist, J. Phys. G 28, R249 (2002).

[20] M. Alford and R. L. Jaffe, Nucl. Phys. B578, 367 (2000).

[21] H. Y. Cheng, C. K. Chua, and K. C. Yang, Phys. Rev. D 73, 014017 (2006).

[22] Y. Li, C. D. Lu, and C. F. Qiao, Phys. Rev. D 73, 094006 (2006).

[23] Y. Li and C. D. Lu, J. Phys. G 29, 2115 (2003); C. D. Lu and K. Ukai, Eur. Phys. J. C 28, 305 (2003); Y. Li and C. D. Lu, High Energy Phys. Nucl. Phys. 27, 1062 (2003).

[24] H. N. Li and B. Tseng, Phys. Rev. D 57, 443 (1998).C. D. Lu and M. Z. Yang, Eur. Phys. J. C 23, 275 (2002).

[25] H.-N. Li, Phys. Rev. D 66, 094010 (2002).

[26] C. H. Chang and H. N. Li, Phys. Rev. D 55, 5577 (1997); T. W. Teh and H. N. Li, Phys. Rev. D 56, 1615 (1997).

[27] G. Bell and T. Feldmann, J. High Energy Phys. 04 (2008) 061; Y. Jia, J. X. Wang, and D. Yang, J. High Energy Phys. 10 (2011) 105; J. F. Cheng, D. S. Du, and C. D. Lu, Eur. Phys. J. C 45, 711 (2006).

[28] T. Huang, AIP Conf. Proc. 68, 1000 (1980); S. J. Brodsky, T. Huang, and G. P. Lepage, in Particles and Fields 2: Proceedings of the Summer Institute, Banff, Canada, edited by A.Z. Capri and A. N. Kamal (Plenum, New York, 1981), p. 143; Z. Dzimbowski, and L. Mankiewicz, Phys. Rev. Lett. 55, 1839 (1985); Z. Dzimbowski, and L. Mankiewicz, Phys. Rev. Lett. 58, 2175 (1987); R. Jakob, P. Kroll, and M. Raulfs, J. Phys. G 22, 45 (1996).

[29] X. H. Guo and T. Huang, Phys. Rev. D 43, 2931 (1991); M. A. G. Nobary and B. Javadi, Eur. Phys. J. C 42, 37 (2005); X. G. Wu, T. Huang, and Z. Y. Fang, Eur. Phys. J. C 52, 561 (2007); Y. Sun, X. G. Wu, F. Zuo, and T. Huang, Eur. Phys. J. C 67, 117 (2010).

[30] X. Liu, H. N. Li, and Z. J. Xiao, arXiv:1801.06145.

[31] T. Kurimoto, H. N. Li, and A. I. Sanda, Phys. Rev. D 67, 054028 (2003); R. H. Li, C. D. Lu, and H. Zou, Phys. Rev. D 78, 014018 (2008); H. Zou, R. H. Li, X. X. Wang, and C. D. Lu, J. Phys. G 37, 015002 (2010); R. H. Li, C. D. Lu, A. I. Sanda, and X. X. Wang, Phys. Rev. D 81, 034006 (2010).

[32] E. Follana, C. T. H. Davies, G. P. Lepage, and J. Shigemitsu (HPQCD and UKQCD Collaborations), Phys. Rev. Lett. 100, 062002 (2008).

[33] H. Y. Cheng, Phys. Rev. D 67, 034024 (2003).

[34] W. Wang, Y. L. Shen, Y. Li, and C. D. Lu, Phys. Rev. D 74, 114010 (2006).

[35] J. W. Li, D. S. Du, and C. D. Lu, Eur. Phys. J. C 72, 2229 (2012); W. Ochs, J. Phys. G 40, 043001 (2013); S. Stone and L. Zhang, Phys. Rev. Lett. 111, 062001 (2013).

[36] R. Aaij et al. (LHCb Collaboration), Phys. Rev. D 87, 052001 (2013).

[37] A. V. Anisovich, V. V. Anisovich, and V. A. Nikonov, Eur. Phys. J. A 12, 103 (2001); Phys. At. Nucl. 65, 497 (2002); A. Gokalp, Y. Sarac, and O. Yilmaz, Phys. Lett. B 609, 291 (2005); R. Fleischer, R. Knegjens, and G. Ricciardi, Eur. Phys. J. C 71, 1832 (2011). 
[38] H. Y. Cheng, C. K. Chua, and K. F. Liu, Phys. Rev. D 74, 094005 (2006).

[39] H. Y. Cheng, C. K. Chua, and K. F. Liu, Phys. Rev. D 92, 094006 (2015); F. Brunner, D. Parganlija, and A. Rebhan, Phys. Rev. D 91, 106002 (2015); S. Janowski, F. Giacosa, and D. H. Rischke, Phys. Rev. D 90, 114005 (2014).

[40] C. D. Lu, Y. M. Wang, and H. Zou, Phys. Rev. D 75, 056001 (2007).
[41] G. Buchalla, A. J. Buras, and M. E. Lautenbacher, Rev. Mod. Phys. 68, 1125 (1996); A. J. Buras, arXiv: hep-ph/9806471.

[42] A. Ali, G. Kramer, and C. D. Lu, Phys. Rev. D 58, 094009 (1998).

[43] R. Aaij et al. (LHCb Collaboration), Phys. Rev. D 92, 032002 (2015); J. High Energy Phys. 08 (2015) 005.

[44] Y. N. Gao, J. B. He, P. Robbe, M.-H. Schune, and Z. W. Yang, Chin. Phys. Lett. 27, 061302 (2010). 